\title{
Apparent horizon and gravitational thermodynamics of the Universe: Solutions to the temperature and entropy confusions, and extensions to modified gravity
}

\author{
David Wenjie Tian* \\ Faculty of Science, Memorial University, St. John's, Newfoundland, Canada, A1C 5S7 \\ Ivan Booth ${ }^{\dagger}$ \\ Department of Mathematics and Statistics, Memorial University, St. John's, Newfoundland, Canada, A1C 5S7
}

\begin{abstract}
The thermodynamics of the Universe is restudied by requiring its compatibility with the holographic-style gravitational equations which govern the dynamics of both the cosmological apparent horizon and the entire Universe, and possible solutions are proposed to the existent confusions regarding the apparent-horizon temperature and the cosmic entropy evolution. We start from the generic Lambda Cold Dark Matter $(\Lambda \mathrm{CDM})$ cosmology of general relativity (GR) to establish a framework for the gravitational thermodynamics. The Cai-Kim Clausius equation $\delta Q=T_{\mathrm{A}} d S_{\mathrm{A}}=-d E_{\mathrm{A}}=-A_{\mathrm{A}} \psi_{t}$ for the isochoric process of an instantaneous apparent horizon indicates that, the Universe and its horizon entropies encode the positive heat out thermodynamic sign convention, which encourages us to adjust the traditional positive-heat-in Gibbs equation into the positive-heat-out version $d E_{m}=-T_{m} d S_{m}-P_{m} d V$. It turns out that the standard and the generalized second laws (GSLs) of nondecreasing entropies are always respected by the event-horizon system as long as the expanding Universe is dominated by nonexotic matter $-1 \leq w_{m} \leq 1$, while for the apparent-horizon simple open system the two second laws hold if $-1 \leq w_{m}<-1 / 3$; also, the artificial local equilibrium assumption is abandoned in the GSL. All constraints regarding entropy evolution are expressed by the equation of state parameter, which show that from a thermodynamic perspective the phantom dark energy is less favored than the cosmological constant and the quintessence. Finally, the whole framework is extended from GR and $\Lambda$ CDM to modified gravities with field equations $R_{\mu \nu}-R g_{\mu \nu} / 2=8 \pi G_{\text {eff }} T_{\mu \nu}^{\text {(eff) }}$. Furthermore, this paper argues that the Cai-Kim temperature is more suitable than Hayward, both temperatures are independent of the inner or outer trappedness of the apparent horizon, and the Bekenstein-Hawking and Wald entropies cannot unconditionally apply to the event and particle horizons.
\end{abstract}

PACS numbers: $04.20 . \mathrm{Cv}, 04.50 . \mathrm{Kd}, 98.80 . \mathrm{Jk}$

\section{INTRODUCTION}

The thermodynamics of the Universe is quite an interesting problem and has attracted a lot of discussion. Pioneering work dates back to the investigations of cosmic entropy evolutions for the spatially flat de Sitter Universe [1] dominated by a positive cosmological constant, while recent studies have covered both the first and second laws of thermodynamics for the Friedmann-Robertson-Walker (FRW) Universe with a generic spatial curvature.

Recent interest on the first law of thermodynamics for the Universe was initiated by Cai and Kim's derivation of the Friedmann equations from a thermodynamic approach [2]: this is actually a continuation of Jacobson's work to recover Einstein's equation from the equilibrium Clausius relation on local Rindler horizons [3], and also a part of the effort to seek the connections between thermodynamics and gravity [4] following the discovery of black hole thermodynamics [5]. For general relativity (GR), Gauss-Bonnet and Lovelock gravities, Akbar and Cai reversed the formulation in [2] by rewriting the Friedmann equations into the heat balance equation and the unified first law of thermodynamics at the cosmological apparent horizon [6]. The method of [6] was soon generalized to other theories of gravity to construct the effec-

\footnotetext{
*wtian@mun.ca

† ibooth@mun.ca
}

tive total energy differentials by the corresponding modified Friedmann equations, such as the scalar-tensor gravity in [7], $f(R)$ gravity in [8], braneworld scenarios in [9, 10], generic $f\left(R, \phi, \nabla_{\alpha} \phi \nabla^{\alpha} \phi\right)$ gravity in [11], and Horava-Lifshitz gravity in [12]. Also, at a more fundamental level, the generic field equations of $F\left(R, \phi,-\frac{1}{2} \nabla_{\alpha} \phi \nabla^{\alpha} \phi, \mathcal{G}\right)$ gravity are recast into the form of Clausius relation in [13].

Besides the first laws on the construction of various energyconservation and heat-transfer equations, the entropy evolution of the Universe has also drawn plenty of attention. However, the cosmic entropies are almost exclusively studied in the generalized rather than the standard second laws [1425]. In fact, investigations via the traditional Gibbs equation $d E_{m}=T_{m} d \tilde{S}_{m}-P_{m} d V$ show that in GR and modified gravities, the evolution of the physical entropy $\tilde{S}_{m}$ for the matter inside the apparent and the event horizons departs dramatically from the desired nondecreasing behaviors; especially that $\tilde{S}_{m}$ inside the future-pointed event horizon always decreases under the dominance of nonexotic matter above the phantom divide. Thus the generalized second law (GSL) has been employed, which adds up $\tilde{S}_{m}$ with the geometrically defined entropy of the cosmological causal boundaries and anticipates the total entropy to be nondecreasing so that the standard second law could be rescued. For example, GSL has been studied in [14] for a flat Universe with multiple entropy sources (thermal, geometric, quantum etc.) by the entropy ansatz $S=|H|^{\alpha}$ $(\alpha>-3)$, in [15] for the event-horizon system of a quintomdominated flat Universe, and [16] for various interacting dark 
energy models.

Moreover, the GSL has also been used as a validity constraint on modified and alternative theories of gravity. For instance, the GSL has been imposed on the event-horizon system of the flat Universe of $f(R)$ gravity in [17], tentatively to the flat apparent-horizon system of generic modified gravities in [18], to the higher-dimensional GaussBonnet and Lovelock gravities in [19], to the Gauss-Bonnet, Randall-Sundrum and Dvali-Gabadadze-Porrati braneworlds in [20], the Horava-Lifshitz gravity in [21], $F(R, \mathcal{G})$ generalized Gauss-Bonnet gravity in [22], $f(T)$ generalized teleparallel gravity in [23], scalar-tensor-chameleon gravity in [24], and the self-interacting $f(R)$ gravity in [25]. Note that in the studies of GSLs, the debatable "local equilibrium assumption" has been widely adopted which supposes that the matter content and the causal boundary in use (mainly the apparent or the event horizon) would have the same temperature [16, 19$22,24,25]$.

Unlike laboratory thermodynamics which is a welldeveloped self-consistent framework, the thermodynamics of the Universe is practically a mixture of ordinary thermodynamics with analogous gravitational quantities, for which the consistency between the first and second laws and among the setups of thermodynamic functions are not yet verified. For example, the Hayward temperature $\kappa / 2 \pi[7,9]$ or $|\kappa| / 2 \pi$ $[8,10]$ which formally resembles the Hawking temperature of (quasi)stationary black holes [5] has been adopted in the first laws, while in GSLs both $|\kappa| / 2 \pi[18-20,24,25]$ and the CaiKim temperature [16, 21, 22] are used. Moreover, in existent literature we have noticed six questions regarding the gravitational thermodynamics of the Universe:

(1) For the Cai-Kim and the Hayward temperatures, which one is more appropriate for the cosmological boundaries? By solving this temperature confusion, the equations of total energy differential at the horizons could also be determined;

(2) For the Bekenstein-Hawking entropy in GR and the Wald entropy in modified gravities, are they unconditionally applicable to both the cosmological apparent and the event horizons?

(3) Is the standard second law for the physical matter really ill-behaved and thus needs to be saved by the GSL? This constitutes the cosmological entropy confusion;

(4) Is the artificial local equilibrium assumption really necessary for the GSL?

(5) The region enveloped by the apparent horizon is actually a thermodynamically open system with the absolute cosmic Hubble flow crossing the horizon; how will this fact influence the entropy evolution?

(6) Are the thermodynamic quantities fully consistent with each other when the cosmic gravitational thermodynamics is systemized?

In this paper, we will try to answer these questions.
This paper is organized as follows. Starting with GR and the $\Lambda$ CDM Universe (where $\Lambda$ denotes generic dark energy), in Sec. II we derive the holographic-style dynamical equations governing the apparent-horizon dynamics and the cosmic spatial expansion, which yield the constraints from the EoS parameter $w_{m}$ on the evolution and metric signature of the apparent horizon. Section III demonstrates how these holographicstyle gravitational equations imply the unified first law of thermodynamics and the Clausius equation, and shows the latter encodes the positive-heat-out sign convention for the horizon entropy. In Sec. IV the Cai-Kim temperature is extensively compared with Hayward, with the former chosen for further usage in Sec. V, where we adjust the traditional Gibbs equation into the Positive Out convention to investigate the entropy evolution for the simple open systems enveloped by the apparent and event horizons. Finally the whole framework of gravitational thermodynamics is extended from $\Lambda$ CDM model and GR to generic modified gravity in Sec. VI. Throughout this paper, we adopt the sign convention $\Gamma_{\beta \gamma}^{\alpha}=\Gamma_{\beta \gamma}^{\alpha}$, $R_{\beta \gamma \delta}^{\alpha}=\partial_{\gamma} \Gamma_{\delta \beta}^{\alpha}-\partial_{\delta} \Gamma_{\gamma \beta}^{\alpha} \cdots$ and $R_{\mu \nu}=R_{\mu \alpha v}^{\alpha}$ with the metric signature $(-,+++)$.

\section{DYNAMICS OF THE COSMOLOGICAL APPARENT HORIZON}

\section{II.1. Apparent horizon and observable Universe}

The FRW metric provides the most general description for the spatially homogeneous and isotropic Universe. In the $(t, r, \theta, \varphi)$ coordinates for an observer comoving with the cosmic Hubble flow, it has the line element (e.g. [2, 26])

$$
\begin{aligned}
d s^{2} & =-d t^{2}+\frac{a(t)^{2}}{1-k r^{2}} d r^{2}+a(t)^{2} r^{2}\left(d \theta^{2}+\sin ^{2} \theta d \varphi^{2}\right) \\
& =h_{\alpha \beta} d x^{\alpha} d x^{\beta}+\Upsilon^{2}\left(d \theta^{2}+\sin ^{2} \theta d \varphi^{2}\right),
\end{aligned}
$$

where $a(t)$ refers to the scale factor to be specified by the gravitational field equations, and the index $k$ denotes the normalized spatial curvature, with $k=\{+1,0,-1\}$ corresponding to closed, flat and open Universes, respectively. $h_{\alpha \beta}:=$ $\operatorname{diag}\left[-1, \frac{a(t)^{2}}{1-k r^{2}}\right]$ represents the transverse two-metric spanned by $x^{\alpha}=(t, r)$, and $\Upsilon:=a(t) r$ stands for the astronomical circumference/areal radius. Based on Eq.(1), one can establish the following null tetrad adapted to the spherical symmetry and the null radial flow,

$$
\begin{aligned}
\ell^{\mu} & =\left(1, \frac{\sqrt{1-k r^{2}}}{a}, 0,0\right) \\
n^{\mu} & =\frac{1}{2}\left(-1, \frac{\sqrt{1-k r^{2}}}{a}, 0,0\right) \\
m^{\mu} & =\frac{1}{\sqrt{2} \Upsilon}\left(0,0,1, \frac{i}{\sin \theta}\right),
\end{aligned}
$$

which has been adjusted to be compatible with the metric signature $(-,+++)$ (e.g. Appendix B in [27]). By calculating the Newman-Penrose spin coefficients $\rho_{\mathrm{NP}}:=-m^{\mu} \bar{m}^{\nu} \nabla_{\nu} \ell_{\mu}$ 
and $\mu_{\mathrm{NP}}:=\bar{m}^{\mu} m^{\nu} \nabla_{\nu} n_{\mu}$, the outward expansion rate $\theta_{(\ell)}=$ $-\left(\rho_{\mathrm{NP}}+\bar{\rho}_{\mathrm{NP}}\right)$ and the inward expansion $\theta_{(n)}=\mu_{\mathrm{NP}}+\bar{\mu}_{\mathrm{NP}}$ are respectively found to be

$$
\begin{aligned}
& \theta_{(\ell)}=2 H+2 \Upsilon^{-1} \sqrt{1-\frac{k \Upsilon^{2}}{a^{2}}} \\
& \theta_{(n)}=-H+\Upsilon^{-1} \sqrt{1-\frac{k \Upsilon^{2}}{a^{2}}}
\end{aligned}
$$

where $H$ refers to the time-dependent Hubble parameter of cosmic spatial expansion, and $H:=\frac{\dot{a}}{a}$ with the overdot denoting the derivative with respect to the comoving time $t$. For the expanding $(H>0)$ Universe, $\theta_{(\ell)}$ and $\theta_{(n)}$ locate the apparent horizon $\Upsilon=\Upsilon_{\mathrm{A}}$ by the unique marginally inner trapped horizon [28] at

$$
\Upsilon_{\mathrm{A}}=\frac{1}{\sqrt{H^{2}+\frac{k}{a^{2}}}}
$$

with $\theta_{(\ell)}=4 H>0, \theta_{(n)}=0$, and also $\partial_{\mu} \Upsilon$ becomes a null vector with $g^{\mu \nu} \partial_{\mu} \Upsilon \partial_{\nu} \Upsilon=0$ at $\Upsilon_{\mathrm{A}}$. Immediately the temporal derivative of Eq.(4) yields the kinematic equation

$$
\dot{\Upsilon}_{\mathrm{A}}=-H \Upsilon_{\mathrm{A}}^{3}\left(\dot{H}-\frac{k}{a^{2}}\right) .
$$

Just like $\Upsilon_{\mathrm{A}}$ and $\dot{\Upsilon}_{\mathrm{A}}$, hereafter quantities evaluated on or related to the apparent horizon will be highlighted by the subscript $A$.

$\left\{\ell^{\mu}, n^{\mu}\right\}$ in Eq.(2) coincide with the outgoing and ingoing tangent vector fields of the null radial congruence that is sent towards infinity by the comoving observer at $r=0$, and ingoing signals from the antitrapped region $\Upsilon>\Upsilon_{\mathrm{A}}$ (where $\left.\theta_{(\ell)}>0, \theta_{(n)}>0\right)$ can no longer cross the marginally inner trapped $\Upsilon_{\mathrm{A}}$ and return to the observer. However, the region $\Upsilon \leq \Upsilon_{\mathrm{A}}$ is not necessarily the standard observable Universe in astronomy where ultrahigh redshift and visually superluminal recession can be detected $[29,30]: \Upsilon_{\mathrm{A}}$ is a future-pointed horizon determined in active measurement by the observer, while the observable Universe is the past-pointed region measured by passive reception of distant signals and thus more related to the past particle horizon.

Note that we are working with the generic FRW metric Eq.(1) which allows for a nontrivial spatial curvature. This is not just for theoretical generality: in fact, astronomical observations indicate that the Universe may not be perfectly flat. For example, in the $o \Lambda \mathrm{CDM}$ sub-model with a strict vacuumenergy condition $w_{\Lambda}=-1$, the nine-years data from the Wilkinson Microwave Anisotropy Probe (WMAP) and other sources like the Baryon Acoustic Oscillations (BAO) yield the fractional energy density $\Omega_{k}=-0.0027_{-0.0038}^{+0.0039}$ [31] for the spatial curvature, independently the time-delay measurements of two strong gravitational lensing systems along with the sevenyears WMAP data find $\Omega_{k}=0.003_{-0.006}^{+0.005}$ [32], while most recently analyses based on BAO data give $\Omega_{k}=-0.003 \pm 0.003$ [33].

\section{II.2. Holographic-style dynamical equations}

The matter content of the Universe is usually portrayed by a perfect-fluid type stress-energy-momentum tensor, and in the metric-independent form it reads

$$
\begin{aligned}
& T_{v}^{\mu(m)}=\operatorname{diag}\left[-\rho_{m}, P_{m}, P_{m}, P_{m}\right] \\
& \text { with } \quad P_{m} / \rho_{m}=: w_{m},
\end{aligned}
$$

where $w_{m}$ refers to the equation of state $(\mathrm{EoS})$ parameter. Substituting this $T_{\mu \nu}^{(m)}$ and the metric Eq.(1) into Einstein's equation $R_{\mu \nu}-\frac{1}{2} R g_{\mu \nu}=8 \pi G T_{\mu \nu}^{(m)}$, one obtains the first and the second Friedmann equations

$$
\begin{gathered}
H^{2}+\frac{k}{a^{2}}=\frac{8 \pi G}{3} \rho_{m} \text { and } \\
\dot{H}-\frac{k}{a^{2}}=-4 \pi G\left(1+w_{m}\right) \rho_{m}=-4 \pi G h_{m} \\
\text { or } 2 \dot{H}+3 H^{2}+\frac{k}{a^{2}}=-8 \pi G P_{m},
\end{gathered}
$$

where $h_{m}=\rho_{m}+P_{m}=\left(1+w_{m}\right) \rho_{m}$ refers to the enthalpy density.

Primarily, the first and second Friedmann equations are respectively the first and second order differential equations of the scale factor $a(t)$, which is the only unspecified function in the metric Eq.(1). On the other hand, recall the location and the time-derivative of the cosmological apparent horizon in Eqs. (4) and (5), and thus Eq.(7) can be rewritten into

$$
\begin{gathered}
\Upsilon_{\mathrm{A}}^{-2}=\frac{8 \pi G}{3} \rho_{m}, \\
\dot{\Upsilon}_{\mathrm{A}}=4 \pi G H \Upsilon_{\mathrm{A}}^{3}\left(1+w_{m}\right) \rho_{m}=4 \pi G H \Upsilon_{\mathrm{A}}^{3} h_{m},
\end{gathered}
$$

which manifest themselves as the dynamical equations of the apparent horizon. However, they also describe the dynamics of spatial expansion for the entire Universe, so for this usage we will dub Eqs.(8) and (9) the "holographic-style" dynamical equations since they reflect the spirit of holography [we are using the word "holographic" in a generic sense as opposed to the standard terminology holographic principle in quantum gravity and string theory [34] or the holographic gravity method [35]].

Eq.(8) immediately implies that, for the late-time Universe dominated by dark energy $\rho_{m}=\rho_{\Lambda}$, the apparent horizon serves as the natural infrared cutoff for the holographic dark energy model [36], in which the dark-energy density $\rho_{\Lambda}^{(\mathrm{HG})}$ relies on the scale of the infrared cutoff $\Upsilon_{\text {IR }}$ by $\rho_{\Lambda}^{(\mathrm{HG})}=$ $3 \Upsilon_{\mathrm{IR}}^{-2} /(8 \pi G)$.

Moreover, with the apparent-horizon area $A_{\mathrm{A}}=4 \pi \Upsilon_{\mathrm{A}}^{2}$, it follows from Eq.(8) that

$$
\rho_{m} A_{\mathrm{A}}=\frac{3}{2 G},
$$


so Eq.(9) can be further simplified into

$$
\dot{\Upsilon}_{\mathrm{A}}=\frac{3}{2} H \Upsilon_{\mathrm{A}}\left(1+w_{m}\right) \text {. }
$$

With the help of Eqs.(8) and (11), for completeness the third member (the $P_{m}$ one) in Eq.(7) can be directly translated into

$$
\Upsilon_{\mathrm{A}}^{-3}\left(\dot{\Upsilon}_{\mathrm{A}}-\frac{3}{2} H \Upsilon_{\mathrm{A}}\right)=4 \pi G H P_{m}
$$

and we keep it in this form without further manipulations for later use in Sec. III.1.

From a mathematical point of view, it might seem trivial to rewrite the Friedmann equations (7) into the holographic-style gravitational equations (8)-(12). However, considering that existent studies on the gravitational thermodynamics of the cosmological apparent horizon always start from the relevant Friedmann equations [6-12, 14-25], we wish that the manipulations of Eq.(7) into Eqs.(8)-(12) could make the formulations physically more meaningful and more concentrative on the horizon $\Upsilon_{\mathrm{A}}$ itself. Also, we will proceed to investigate some useful properties of the apparent horizon as necessary preparations for the horizon thermodynamics.

Eq.(11) clearly shows that, for an expanding Universe $(H>0)$ the apparent-horizon radius $\Upsilon_{\mathrm{A}}$ can be either expanding, contracting or even static, depending on the domain of the EoS parameter $w_{m}$ or equivalently the sign of the enthalpy density $h_{m}$. In the $\Lambda \mathrm{CDM}$ cosmology, $\rho_{m}$ could be decomposed into all possible components, $\rho_{m}=\sum \rho_{m}^{(i)}=$ $\rho_{m}($ baryon $)+\rho_{m}($ radiation $)+\rho_{m}($ neutrino $)+\rho_{m}($ dark matter $)+$ $\rho_{m}$ (dark energy) $+\cdots$, and the same for $P_{m}$. In principle there should be an EoS parameter $w_{m}^{(i)}=P_{m}^{(i)} / \rho_{m}^{(i)}$ associated to each energy component. However, practically we can regard $w_{m}$ either as that of the absolutely dominating matter, or the weighted average for all relatively dominating components

$$
w_{m}=\frac{\sum P_{m}^{(i)}}{\rho_{m}}=\frac{\sum w_{m}^{(i)} \rho_{m}^{(i)}}{\rho_{m}}=\sum \alpha_{i} w_{m}^{(i)},
$$

with the weight coefficient given by $\alpha_{i}=\rho_{m}^{(i)} / \rho_{m}$, and thus $w_{m}$ varies over cosmic time scale. Then it follows from Eq.(11) that:

\begin{tabular}{|c|c|c|c|}
\hline$w_{m}$ & dominating matter & enthalpy density & $\dot{\Upsilon}_{\mathrm{A}}$ \\
\hline \hline $\begin{array}{c}-1 / 3 \leq w_{m}(\leq 1) \text { and } \\
-1<w_{m}<-1 / 3\end{array}$ & $\begin{array}{c}\text { ordinary matter, and } \\
\text { quintessence [37] }\end{array}$ & $h_{m}>0$ & $\dot{\Upsilon}_{\mathrm{A}}>0$, expanding \\
\hline$w_{m}=-1$ & $\begin{array}{c}\text { cosmological constant or } \\
\text { vacuum energy [38] }\end{array}$ & $h_{m}=0$ & $\dot{\Upsilon}_{\mathrm{A}}=0$, static \\
\hline$w_{m}<-1$ & phantom [39] & $h_{m}<0$ & $\dot{\Upsilon}_{\mathrm{A}}<0$, contracting \\
\hline
\end{tabular}

The dominant energy condition [40] $\rho_{m} \geq\left|P_{m}\right|$ imposes the constraint $-1 \leq w_{m} \leq 1$ for nonexotic matter. Here we retain the upper limit $w_{m} \leq 1$ but loosen the lower limit, allowing $w_{m}$ to cross the barrier $w_{m}=-1$ into the exotic phantom domain $w_{m}<-1$. The upper limit however is bracketed as $(\leq 1)$ to indicate that it is a physical rather than mathematical constraint.

\section{II.3. Induced metric of the apparent horizon}

The total derivative of $\Upsilon=\Upsilon(t, r)$ yields $a d r=d \Upsilon-H \Upsilon d t$, which recasts the FRW line element Eq.(1) into the $(t, \Upsilon, \theta, \varphi)$ coordinates as

$$
\begin{gathered}
d s^{2}=\left(1-\frac{k \Upsilon^{2}}{a^{2}}\right)^{-1}\left(-\left(1-\frac{\Upsilon^{2}}{\Upsilon_{\mathrm{A}}^{2}}\right) d t^{2}-2 H \Upsilon d t d \Upsilon+d \Upsilon^{2}\right) \\
+\Upsilon^{2}\left(d \theta^{2}+\sin ^{2} \theta d \varphi^{2}\right) .
\end{gathered}
$$

Although the comoving transverse coordinates $(t, r)$ are easier to work with, we will switch to the more physical coordinates $(t, \Upsilon)$ whenever necessary. The metric Eq.(14) reduces to become a three-dimensional hypersurface in the $(t, \theta, \varphi)$ coordinates at the apparent horizon $\Upsilon_{\mathrm{A}}=\Upsilon_{\mathrm{A}}(t)$, and with Eq.(11), the induced horizon metric turns out to be

$$
\begin{aligned}
d s^{2} & =\left(H \Upsilon_{\mathrm{A}}\right)^{-2}\left(\dot{\Upsilon}_{\mathrm{A}}-2 H \Upsilon_{\mathrm{A}}\right) \dot{\Upsilon}_{\mathrm{A}} d t^{2}+\Upsilon_{\mathrm{A}}^{2}\left(d \theta^{2}+\sin ^{2} \theta d \varphi^{2}\right) \\
& =\frac{9}{4}\left(w_{m}+1\right)\left(w_{m}-\frac{1}{3}\right) d t^{2}+\Upsilon_{\mathrm{A}}^{2}\left(d \theta^{2}+\sin ^{2} \theta d \varphi^{2}\right)
\end{aligned}
$$

Here $w_{m}$ shows up in the coefficients of $d t^{2}$, and indeed the spirit of geometrodynamics allows and encourages physical parameters to directly participate in the spacetime metric, just like the mass, electric charge and angular momentum parameters in the Kerr-Newmann solution. It is easily seen that the signature of the apparent horizon solely relies on the domain of $w_{m}$ regardless of the Universe being expanding or contracting.

(1) For $-1<w_{m}<1 / 3$, the apparent horizon $\Upsilon_{\mathrm{A}}$ has the signature $(-,++)$ and is timelike, which shares the signature of a quasilocal timelike membrane in black-hole physics [28, 41].

(2) For $w_{m}<-1$ or $1 / 3<w_{m}(\leq 1)$, the signature is $(+,++)$ and thus $\Upsilon_{\mathrm{A}}$ is spacelike. This situation has the same signature with the dynamical black-hole horizons [42].

(3) For $w_{m}=-1$ or $w_{m}=1 / 3, \Upsilon_{\mathrm{A}}$ is a null surface with the signature $(0,++)$, so it coincides with the cosmologi- 
cal event horizon $\Upsilon_{\mathrm{E}}:=a \int_{t}^{\infty} a^{-1} d \hat{t}[26,43]$ which by definition is a future-pointed null causal boundary, and it shares the signature of isolated black-hole horizons [27].

Note that these analogies between $\Upsilon_{\mathrm{A}}$ and black-hole horizons are limited to the metric signature, while the behaviors of their expansions $\left\{\theta_{(\ell)}, \theta_{(n)}\right\}$ and the horizon trappedness are entirely different. Among the two critical values, $w_{m}=-1$ corresponds to the de Sitter Universe dominated by a positive cosmological constant (or vacuum energy) [1], while $w_{m}=1 / 3$ refers to the highly relativistic limit of $w_{m}$ and the EoS of radiation, with the trace of the the stress-energy-momentum tensor $g^{\mu v} T_{\mu \nu}^{(m)}=\left(3 w_{m}-1\right) \rho_{m}$ vanishing at $w_{m}=1 / 3$. As will be shown later in Sec. IV, $w_{m}=1 / 3$ also serves as the "zero temperature divide" if the apparent-horizon temperature were measured by $\kappa / 2 \pi$ in terms of the Hayward surface gravity $\kappa$.

\section{II.4. Relative evolution equations}

The nontrivial $t$-component of $\nabla_{\mu} T_{v}^{\mu(m)}=0$ with respect to the metric Eq.(1) leads to the continuity equation for the cosmic perfect fluid

$$
\dot{\rho}_{m}+3 H\left(1+w_{m}\right) \rho_{m}=0 .
$$

Thus for the relative evolution rate of the energy density $\dot{\rho}_{m} / \rho_{m}$, its ratio over that of the cosmic scale factor $\dot{a} / a=H$ synchronizes with the instantaneous value of the EoS parameter, $\frac{\dot{\rho}_{m}}{\rho_{m}} / \frac{\dot{a}}{a}=-3\left(1+w_{m}\right)$. This relation is not alone, as one could easily observe from Eq.(11) that the relative evolution rate of the apparent-horizon radius $\dot{\Upsilon}_{\mathrm{A}} / \Upsilon_{\mathrm{A}}$ is normalized by $\dot{a} / a$ into $\frac{\dot{\Upsilon}_{\mathrm{A}}}{\Upsilon_{\mathrm{A}}} / \frac{\dot{a}}{a}=\frac{3}{2}\left(1+w_{m}\right)$. These two equations reveal the interesting result that throughout the history of the Universe, the relative evolution rate of the energy density is always proportional to that of the apparent-horizon radius:

$$
\frac{\dot{\rho}_{m}}{\rho_{m}} / \frac{\dot{\Upsilon}_{\mathrm{A}}}{\Upsilon_{\mathrm{A}}}=-2 .
$$

In fact, integration of Eq.(17) yields $\ln \rho_{m} \propto-2 \ln \Upsilon_{\mathrm{A}}$ and thus $\rho_{m} \propto \Upsilon_{\mathrm{A}}^{-2}$, which matches the holographic-style dynamical equation (8) with the proportionality constant identified as $\frac{3}{8 \pi G}$.

\section{THERMODYNAMIC IMPLICATIONS OF THE HOLOGRAPHIC-STYLE DYNAMICAL EQUATIONS}

In Sec. II, based on Eqs.(8)-(12) we have analyzed some properties of the cosmological apparent horizon $\Upsilon_{\mathrm{A}}$ to facilitate the subsequent discussion; one can refer to [43] for more discussion of the horizon $\Upsilon_{\mathrm{A}}$. From this section on, we will continue to investigate the thermodynamic implications of the holographic-style gravitational equations (8)-(12).

\section{III.1. Unified first law of thermodynamics}

The mass $M=\rho_{m} V$ of cosmic fluid within a sphere of radius $\Upsilon$, surface area $A=4 \pi \Upsilon^{2}$ and volume $V=\frac{4}{3} \pi \Upsilon^{3}$, can be geometrically recovered from the spacetime metric and we will identify it as the total internal energy $E$. With the MisnerSharp mass/energy [44] $E_{\mathrm{MS}}:=\frac{\Upsilon}{2 G}\left(1-h^{\alpha \beta} \partial_{\alpha} \Upsilon \partial_{\beta} \Upsilon\right)$ for spherically symmetric spacetimes, Eq.(1) with $h^{\alpha \beta}=\operatorname{diag}\left[-1, \frac{a^{2}}{1-k r^{2}}\right]$ for the Universe yields

$$
E=\frac{\Upsilon^{3}}{2 G \Upsilon_{\mathrm{A}}^{2}},
$$

and its equivalence with the physically defined mass $E=$ $M=\rho_{m} V$ is guaranteed by Eq.(8). Equation (18) can also be reconstructed in the tetrad Eq.(2) from the Hawking energy [45] $E_{\mathrm{Hk}}:=\frac{1}{4 \pi G}\left(\int \frac{d A}{4 \pi}\right)^{1 / 2} \int\left(-\Psi_{2}-\sigma_{\mathrm{NP}} \lambda_{\mathrm{NP}}+\Phi_{11}+\right.$ $\left.\Lambda_{\mathrm{NP}}\right) d A \equiv \frac{1}{4 \pi G}\left(\int \frac{d A}{4 \pi}\right)^{1 / 2}\left(2 \pi-\int \rho_{\mathrm{NP}} \mu_{\mathrm{NP}} d A\right)$ for twist-free spacetimes. Immediately, the total derivative or transverse gradient of $E=E(t, r)$ is

$$
\begin{aligned}
d E & =-\frac{1}{G} \frac{\Upsilon^{3}}{\Upsilon_{\mathrm{A}}^{3}}\left(\dot{\Upsilon}_{\mathrm{A}}-\frac{3}{2} H \Upsilon_{\mathrm{A}}\right) d t+\frac{3}{2 G} \frac{\Upsilon^{2}}{\Upsilon_{\mathrm{A}}^{2}} a d r \\
& =-\frac{\dot{\Upsilon}_{\mathrm{A}}}{G} \frac{\Upsilon^{3}}{\Upsilon_{\mathrm{A}}^{3}} d t+\frac{3}{2 G} \frac{\Upsilon^{2}}{\Upsilon_{\mathrm{A}}^{2}} d \Upsilon,
\end{aligned}
$$

where the relation $a d r=d \Upsilon-H \Upsilon d t$ has been employed to rewrite Eq.(19) into Eq.(20), with the transverse coordinates from $(t, r)$ to $(t, \Upsilon)$. According to the holographic-style dynamical equations (8), (9) and (12), the energy differentials Eqs.(19) and (20) can be rewritten into

$$
\begin{aligned}
d E & =-A \Upsilon H P_{m} d t+A \rho_{m} a d r \\
& =-A\left(1+w_{m}\right) \rho_{m} H \Upsilon d t+A \rho_{m} d \Upsilon .
\end{aligned}
$$

Eqs.(21) and (22) can be formally compactified into

$$
d E=A \psi+W d V
$$

where $\psi$ and $W$ are respectively the energy supply covector

$$
\begin{aligned}
\psi & =-\frac{1}{2} \rho_{m}\left(1+w_{m}\right) H \Upsilon d t+\frac{1}{2} \rho_{m}\left(1+w_{m}\right) a d r \\
& =-\rho_{m}\left(1+w_{m}\right) H \Upsilon d t+\frac{1}{2} \rho_{m}\left(1+w_{m}\right) d \Upsilon,
\end{aligned}
$$

and the work density

$$
W=\frac{1}{2}\left(1-w_{m}\right) \rho_{m} .
$$

Eq.(23) is exactly the unified first law of (equilibrium) thermodynamics proposed by Hayward [46], and one can see from the derivation process that it applies to a volume of arbitrary areal radius $\Upsilon$, no matter $\Upsilon<\Upsilon_{\mathrm{A}}, \Upsilon=\Upsilon_{\mathrm{A}}$ or $\Upsilon>\Upsilon_{\mathrm{A}}$. Moreover, $W$ and $\psi$ can respectively be traced back to the scalar invariant $W:=-\frac{1}{2} T_{(m)}^{\alpha \beta} h_{\alpha \beta}$ and the covector invariant $\psi_{\alpha}:=T_{\alpha(m)}^{\beta} \partial_{\beta} \Upsilon+W \partial_{\alpha} \Upsilon[46]$, which are valid for all spheri- 
cally symmetric spacetimes besides FRW, and have Eqs.(24), (25) and (26) as their concrete components with respect to the metric Eq.(1).

Note that the "unified" first law Eq.(23) for the gravitational thermodynamics of the Universe is totally different from the first laws in black-hole thermodynamics which balance the energy differential with the first-order variations of the ArnowittDeser-Misner type quantities (such as mass, electric charge, and angular momentum). Instead, Eq.(23) is more related to the geometrical aspects of the thermodynamics-gravity correspondence.

\section{III.2. Clausius equation on the apparent horizon for an isochoric process}

Having seen that the full set of holographic-style dynamical equations (8), (9) and (12) yield the unified first law $d E=$ $A \psi+W d V$ for an arbitrary region in the FRW Universe, we will focus on the volume enclosed by the apparent horizon $\Upsilon_{\mathrm{A}}$. Firstly, Eq.(9) leads to

$$
\frac{\dot{\Upsilon}_{\mathrm{A}}}{G} d t=A_{\mathrm{A}}\left(1+w_{m}\right) \rho_{m} H \Upsilon_{\mathrm{A}} d t
$$

and the left hand side can be manipulated into

$$
\frac{\dot{\Upsilon}_{\mathrm{A}}}{G} d t=\frac{1}{2 \pi \Upsilon_{\mathrm{A}}}\left(\frac{2 \pi \Upsilon_{\mathrm{A}} \dot{\Upsilon}_{\mathrm{A}}}{G} d t\right)=\frac{1}{2 \pi \Upsilon_{\mathrm{A}}} \frac{d}{d t}\left(\frac{\pi \Upsilon_{\mathrm{A}}^{2}}{G}\right)
$$

Applying the geometrically defined Hawking-Bekenstein entropy [5] (in the units $\hbar=c=k$ [Boltzmann] = 1) to the apparent horizon

$$
S_{\mathrm{A}}=\frac{\pi \Upsilon_{\mathrm{A}}^{2}}{G}=\frac{A_{\mathrm{A}}}{4 G}
$$

then employing the Cai-Kim temperature [2, 47]

$$
T_{\mathrm{A}} \equiv \frac{1}{2 \pi \Upsilon_{\mathrm{A}}}
$$

thus $T_{\mathrm{A}} d S_{\mathrm{A}}=\dot{\Upsilon}_{\mathrm{A}} / G d t$ and Eq.(28) can be rewritten into

$$
T_{\mathrm{A}} d S_{\mathrm{A}}=\delta Q_{\mathrm{A}}=-A_{\mathrm{A}} \psi_{t}=-\left.d E_{\mathrm{A}}\right|_{d \Upsilon=0},
$$

where $\psi_{t}$ is the $t$-component of the energy supply covector $\boldsymbol{\psi}=\psi_{t}+\psi_{\Upsilon}=\psi_{\alpha} d x^{\alpha}$ in Eq.(25). This basically reverses Cai and Kim's formulation in [2], and differs from [6] by the setup of the horizon temperature. Eq.(31) is actually the Clausius equation for equilibrium and reversible thermodynamic processes, and the meaning of reversibility compatible with the cosmic dynamics is clarified in Appendix A. Comparing Eq.(31) with the unified first law Eq.(23), one could find that Eq.(31) is just Eq.(23) with the two $d \Upsilon$ components removed and then evaluated at $\Upsilon_{\mathrm{A}}$. Assuming that the apparent horizon locates at $\Upsilon_{\mathrm{A} 0} \equiv \Upsilon_{\mathrm{A}}\left(t=t_{0}\right)$ at an arbitrary moment $t_{0}$, then during the infinitesimal time interval $d t$ the horizon will move to $\Upsilon_{\mathrm{A} 0}+\dot{\Upsilon}_{\mathrm{A} 0} d t$; meanwhile, for the isochoric process of the volume $V\left(\Upsilon_{\mathrm{A} 0}\right)$ (i.e. a "controlled volume"), the amount of energy across the horizon $\Upsilon_{\mathrm{A} 0}$ is just $d E_{\mathrm{A}}=A_{\mathrm{A} 0} \psi_{t}$ evaluated at $t_{0}$, and for brevity we will drop the subscript " 0 " whenever possible as $t_{0}$ is arbitrary.

The energy-balance equation (31) implies that the region $\Upsilon \leq \Upsilon_{\mathrm{A}}$ enveloped by the cosmological apparent horizon is thermodynamically an open system which exchanges both heat and matter (condensed components in the Hubble flow) with its surroundings/reservoir $\Upsilon \geq \Upsilon_{\mathrm{A}}$. Here we emphasize again that $\Upsilon_{\mathrm{A}}$ is simply a visual boundary preventing ingoing null radial signals from reaching the comoving observer, and the absolute cosmic Hubble flow can still cross $\Upsilon_{\mathrm{A}}$. Also, unlike nonrelativistic thermodynamics in which $\delta Q$ exclusively refers to the heat transfer (i.e. electromagnetic flow), the $\delta Q_{\mathrm{A}}$ in Eq.(31) is used in a mass-energy-equivalence sense and denotes the Hubble energy flow which generally contains different matter components.

Finally, for the open system enveloped by $\Upsilon_{\mathrm{A}}$, we combine the Clausius equation (31) and the unified first law Eq.(23) into the total energy differential

$$
\begin{aligned}
d E_{\mathrm{A}} & =A_{\mathrm{A}} \psi_{t} d t+A_{\mathrm{A}}(\psi \Upsilon+W) d \Upsilon_{\mathrm{A}} \\
& =-T_{\mathrm{A}} d S_{\mathrm{A}}+\rho_{m} A_{\mathrm{A}} d \Upsilon_{\mathrm{A}} \\
& =-T_{\mathrm{A}} d S_{\mathrm{A}}+\rho_{m} d V_{\mathrm{A}} .
\end{aligned}
$$

In fact, by the continuity equation (16) one can verify $-T_{\mathrm{A}} d S_{\mathrm{A}}=V_{\mathrm{A}} d \rho_{m}$, which agrees with the thermodynamic connotation that the heat $-T_{\mathrm{A}} d S_{\mathrm{A}}=\delta Q_{\mathrm{A}}$ measures the loss of internal energy that can no longer be used to do work. In this sense, one may further regard $d E_{\mathrm{A}}+T_{\mathrm{A}} d S_{\mathrm{A}}$ to play the role of the relativistic differential Helmholtz free energy $d \mathbb{F}_{\mathrm{A}}$ for the instantaneous $\Upsilon_{\mathrm{A} 0}$ of temperature $T_{\mathrm{A} 0}$,

$$
d \mathbb{F}_{\mathrm{A}}:=d E_{\mathrm{A}}+T_{\mathrm{A}} d S_{\mathrm{A}}=\rho_{m} d V_{\mathrm{A}}=(\psi \Upsilon+W) d V_{\mathrm{A}},
$$

which represents the maximal work element that can be extracted from the interior of $\Upsilon_{\mathrm{A} 0}$; one could also identify the relativistic differential Gibbs free energy $d \mathbb{G}_{\mathrm{A}}$, which means the "useful" work element, as

$$
d \mathbb{G}_{\mathrm{A}}:=d E_{\mathrm{A}}+T_{\mathrm{A}} d S_{\mathrm{A}}+P_{m} d V_{\mathrm{A}}=\rho_{m}\left(1+w_{m}\right) d V_{\mathrm{A}} .
$$

Note that $d \mathbb{F}_{\mathrm{A}}$ and $d \mathbb{G}_{\mathrm{A}}$ contain $+T_{\mathrm{A}} d S_{\mathrm{A}}$ with a plus instead of a minus sign, because the Cai-Kim Clausius relation $d E_{\mathrm{A}}=-T_{\mathrm{A}} d S_{\mathrm{A}}$ encodes that the horizon entropy $S_{\mathrm{A}}$ is defined in a "positive heat out" rather than the traditional positive-heat-in thermodynamic sign convention, as will be extensively discussed in Sec. V.1.

\section{SOLUTION TO THE HORIZON-TEMPERATURE CONFUSION}

\section{IV.1. The horizon-temperature confusion}

In the thermodynamics of (quasi)stationary black holes [5], the Hawking temperature satisfies $T=\tilde{\kappa} /(2 \pi)$ based on the traditional Killing surface gravity $\tilde{K}$ and the Killing genera- 
tors of the horizon. For the FRW Universe, one has the Hayward inaffinity parameter $\kappa$ [46] in place of the Killing inaffinity, which yields the Hayward surface gravity on the apparent horizon,

$$
\begin{aligned}
\kappa & :=\frac{1}{2} h^{\alpha \beta} \nabla_{\alpha} \nabla_{\beta} \Upsilon=\frac{1}{2 \sqrt{-h}} \partial_{\alpha}\left(\sqrt{-h} h^{\alpha \beta} \partial_{\beta} \Upsilon\right) \\
& \equiv-\frac{\Upsilon}{\Upsilon_{\mathrm{A}}^{2}}\left(1-\frac{\dot{\Upsilon}_{\mathrm{A}}}{2 H \Upsilon_{\mathrm{A}}}\right)=-\left.\frac{1}{\Upsilon_{\mathrm{A}}}\left(1-\frac{\dot{\Upsilon}_{\mathrm{A}}}{2 H \Upsilon_{\mathrm{A}}}\right)\right|_{\Upsilon_{\mathrm{A}}},
\end{aligned}
$$

where $h_{\alpha \beta}=\operatorname{diag}\left[-1, \frac{a^{2}}{1-k r^{2}}\right]$ refers to the transverse twometric in Eq.(1). Then formally following the Hawking temperature, the Hayward temperature of the apparent horizon $\Upsilon_{A}$ is defined either by $[7,9]$

$$
\mathcal{T}_{\mathrm{A}}:=\frac{\kappa}{2 \pi}=-\frac{1}{2 \pi \Upsilon_{\mathrm{A}}}\left(1-\frac{\dot{\Upsilon}_{\mathrm{A}}}{2 H \Upsilon_{\mathrm{A}}}\right)
$$

or $[8,10,18-20,24,25]$

$$
\mathcal{T}_{\mathrm{A}}^{(+)}:=\frac{(\kappa \mid}{2 \pi}=\frac{1}{2 \pi \Upsilon_{\mathrm{A}}}\left(1-\frac{\dot{\Upsilon}_{\mathrm{A}}}{2 H \Upsilon_{\mathrm{A}}}\right),
$$

where we use the symbol $(\kappa \mid$ to denote the partial absolute value of $\kappa$, because existing papers have a priori abandoned the possibility of $\dot{\Upsilon}_{\mathrm{A}} /\left(2 H \Upsilon_{\mathrm{A}}\right) \geq 1$ for $\mathcal{T}_{\mathrm{A}}^{(+)}$. Equation (37) is always supplemented by the assumption [8, 10, 18-20, 24, 25]

$$
\frac{\dot{\Upsilon}_{\mathrm{A}}}{2 H \Upsilon_{\mathrm{A}}}<1
$$

to guarantee a positive $\mathcal{T}_{\mathrm{A}}^{(+)}$which is required by the third law of thermodynamics, and even the condition [18]

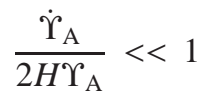

so that $\mathcal{T}_{\mathrm{A}}^{(+)}$can be approximated into the Cai-Kim temperature $[2,47]$

$$
\mathcal{T}_{\mathrm{A}}^{(+)} \approx \frac{1}{2 \pi \Upsilon_{\mathrm{A}}}=T_{\mathrm{A}}
$$

Historically the inverse problem "from thermodynamics to gravitational equations for the Universe" [2] was formulated earlier, in which the Cai-Kim temperature works perfectly for all theories of gravity. Later on, the problem "from FRW gravitational equations to thermodynamics" [6-8] (as the logic in this paper) came into attention in which the Hayward temperature seems to become effective. Considering that two different temperatures make the two mutually inverse problems asymmetric, attempts have been made to reduce the differences between them, mainly the assumptions Eqs.(38) and (39).

Note that when the conditions Eqs.(39) and (40) are applied to Eq.(36), $\mathcal{T}_{\mathrm{A}}$ would become a negative temperature. [7] has suggested that it might be possible to understand this phenomenon as a consequence of the cosmological apparent horizon being inner trapped $\left[\theta_{(\ell)}>0, \theta_{(n)}=0\right]$, as opposed to the positive temperatures of black-hole apparent horizons which are always marginally outer trapped $\left[\theta_{(\ell)}=0, \theta_{(n)}<0\right]$. However, this proposal turns out to be inappropriate; as will be shown at the end of Sec. IV.3, the signs of $\mathcal{T}_{\mathrm{A}}$ actually keep pace with the metric signatures rather than the inner/outer trappedness of the horizon $\Upsilon_{\mathrm{A}}$.

\section{IV.2. Effects of $\mathcal{T}_{\mathbf{A}} d S_{\mathbf{A}}$ and $\mathcal{T}_{\mathbf{A}}^{(+)} d S_{\mathbf{A}}$}

In Sec. III.2, we have seen $T_{\mathrm{A}} d S_{\mathrm{A}}=A_{\mathrm{A}} \psi_{t}$ for the Cai-Kim $T_{\mathrm{A}}=1 /\left(2 \pi \Upsilon_{\mathrm{A}}\right)$, and now let's examine the effects of $\mathcal{T}_{\mathrm{A}}$ and $\mathcal{T}_{\mathrm{A}}^{(+)}$. Given the Bekenstein-Hawking entropy $S_{\mathrm{A}}=A_{\mathrm{A}} / 4 G$, the dynamical equation $\dot{\Upsilon}_{\mathrm{A}}=A_{\mathrm{A}} H \Upsilon_{\mathrm{A}} G\left(1+w_{m}\right) \rho_{m}$ and the energy supply covector $\psi=\psi_{t}+\psi_{\Upsilon}=-\left(1+w_{m}\right) \rho_{m} H \Upsilon_{\mathrm{A}} d t+$ $\frac{1}{2}\left(1+w_{m}\right) \rho_{m} d \Upsilon_{\mathrm{A}}$, one has

$$
\begin{aligned}
\mathcal{T}_{\mathrm{A}} d S_{\mathrm{A}} & =-\frac{\dot{\Upsilon}_{\mathrm{A}}}{G}+\frac{\dot{\Upsilon}_{\mathrm{A}}}{2 G H \Upsilon_{\mathrm{A}}} \dot{\Upsilon}_{\mathrm{A}} d t \\
& =-A_{\mathrm{A}} H \Upsilon_{\mathrm{A}}\left(1+w_{m}\right) \rho_{m} d t+\frac{1}{2} A_{\mathrm{A}}\left(1+w_{m}\right) \rho_{m} d \Upsilon_{\mathrm{A}} \\
& =A_{\mathrm{A}} \psi_{t}+A_{\mathrm{A}} \psi_{\Upsilon}=A_{\mathrm{A}} \psi .
\end{aligned}
$$

Similarly, for the $\mathcal{T}_{\mathrm{A}}^{(+)}$defined in Eq.(37),

$$
\mathcal{T}_{\mathrm{A}}^{(+)} d S_{\mathrm{A}}=-\left(A_{\mathrm{A}} \psi_{t}+A_{\mathrm{A}} \psi_{\Upsilon}\right)=-A_{\mathrm{A}} \psi
$$

Hence, for the two terms comprising $\mathcal{T}_{\mathrm{A}}$ and $\mathcal{T}_{\mathrm{A}}^{(+)}$, the $\pm \frac{1}{2 \pi \Upsilon_{\mathrm{A}}} d S_{\mathrm{A}}$ is balanced by $\mp A_{\mathrm{A}} \psi_{t}$, while the $\pm \frac{\dot{\Upsilon}_{\mathrm{A}}}{2 H \Upsilon_{\mathrm{A}}} d S_{\mathrm{A}}$ is equal to $\pm A_{\mathrm{A}} \psi_{\Upsilon}$. As obtained in e.g. [6]-[12], for the open system enveloped by the cosmological apparent horizon, combining Eqs.(41) and (42) with the unified first law Eq.(23) leads to the total energy differential

$$
\begin{aligned}
d E_{\mathrm{A}} & =\mathcal{T}_{\mathrm{A}} d S_{\mathrm{A}}+W d V_{\mathrm{A}} \\
& =-\mathcal{T}_{\mathrm{A}}^{(+)} d S_{\mathrm{A}}+W d V_{\mathrm{A}},
\end{aligned}
$$

as opposed to $d E_{\mathrm{A}}=-T_{\mathrm{A}} d S_{\mathrm{A}}+\rho_{m} d V_{\mathrm{A}}$ for the Cai-Kim $T_{\mathrm{A}}$.

\section{IV.3. "Zero temperature divide" $w_{m}=1 / 3$ and preference of Cai-Kim temperature}

Now apply the dynamical equation (11) to $\left\{\mathcal{T}_{\mathrm{A}}, \mathcal{T}_{\mathrm{A}}^{(+)}\right\}$and the assumptions in Eqs.(38) and (39). With $\dot{\Upsilon}_{\mathrm{A}}=\frac{3}{2} H \Upsilon_{\mathrm{A}}(1+$ $\left.w_{m}\right)$, the Hayward surface gravity becomes

$$
\kappa=-\frac{1}{\Upsilon_{\mathrm{A}}}\left(1-\frac{\dot{\Upsilon}_{\mathrm{A}}}{2 H \Upsilon_{\mathrm{A}}}\right)=-\frac{3}{4 \Upsilon_{\mathrm{A}}}\left(\frac{1}{3}-w_{m}\right),
$$


so it follows that

$$
\left\{\begin{array}{ll}
w_{m}>\frac{1}{3}: & \kappa>0, \quad|\kappa|=\frac{3}{4 \Upsilon_{\mathrm{A}}}\left(w_{m}-\frac{1}{3}\right) \\
w_{m}=\frac{1}{3}: & \kappa=|\kappa|=0 \\
w_{m}<\frac{1}{3}: & \kappa<0, \quad|\kappa|=\frac{3}{4 \Upsilon_{\mathrm{A}}}\left(\frac{1}{3}-w_{m}\right)
\end{array} .\right.
$$

The Hayward temperature $\mathcal{T}_{\mathrm{A}}$ in Eq.(36) and its partially absolute value $\mathcal{T}_{\mathrm{A}}^{(+)}$in Eq.(37) become

$$
\begin{aligned}
\mathcal{T}_{\mathrm{A}} & =-\frac{3}{8 \pi \Upsilon_{\mathrm{A}}}\left(\frac{1}{3}-w_{m}\right)=-\frac{1}{4} T_{\mathrm{A}}\left(1-3 w_{m}\right) \\
\mathcal{T}_{\mathrm{A}}^{(+)} & =\frac{3}{8 \pi \Upsilon_{\mathrm{A}}}\left(\frac{1}{3}-w_{m}\right)=\frac{1}{4} T_{\mathrm{A}}\left(1-3 w_{m}\right) .
\end{aligned}
$$

Fortunately $\mathcal{T}_{\mathrm{A}}$ and $\mathcal{T}_{\mathrm{A}}^{(+)}$remain as state functions, although Eqs.(36) and (37) carry $\left\{\dot{\Upsilon}_{\mathrm{A}}, H\right\}$ and look like process quantities (see Appendix A for more discussion). Moreover, the supplementary assumption Eq.(38) for $\mathcal{T}_{\mathrm{A}}^{(+)}>0$ turns out to be

$$
\frac{\dot{\Upsilon}_{\mathrm{A}}}{2 H \Upsilon_{\mathrm{A}}}=\frac{3}{4}\left(1+w_{m}\right)<1 \Rightarrow w_{m}<1 / 3
$$

Thus the condition $\frac{\dot{\Upsilon}_{\mathrm{A}}}{2 H \Upsilon_{\mathrm{A}}} \ll 1$ in Eq.(39) could be directly translated into $w_{m} \ll 1 / 3$, which is however inaccurate: in fact, if directly starting from Eq.(46), the approximation $\mathcal{T}_{\mathrm{A}}^{(+)} \approx T_{\mathrm{A}}=1 /\left(2 \pi \Upsilon_{\mathrm{A}}\right)$ will require

$$
w_{m} \rightarrow-1
$$

It is neither mathematically nor physically identical with $w_{m} \ll 1 / 3$ which could only be perfectly satisfied for $w_{m} \rightarrow$ $-\infty$ in the extreme phantom domain.

Eqs.(44) - (48) have rewritten and simplified the original expressions of the Hayward temperatures $\left\{\mathcal{T}_{\mathrm{A}}, \mathcal{T}_{\mathrm{A}}^{(+)}\right\}$in Eqs. $(36,37)$ and their supplementary conditions Eqs. $(38,39)$. Based on these results we realize that it becomes possible to make an extensive comparison between $\left\{\mathcal{T}_{\mathrm{A}}, \mathcal{T}_{\mathrm{A}}^{(+)}\right\}$and the Cai-Kim $T_{\mathrm{A}}=1 /\left(2 \pi \Upsilon_{\mathrm{A}}\right)$, which reveals the following facts.

(1) $\mathcal{T}_{\mathrm{A}}$ is negative definite for $1 / 3<w_{m}(\leq 1)$, positive definite for $w_{m}<1 / 3$, and $\mathcal{T}_{\mathrm{A}} \equiv 0$ for $w_{m}=1 / 3$. We will dub the special value $w_{m}=1 / 3$ as the Hayward "zero temperature divide", which is inspired by the terminology "phantom divide" for $w_{m}=-1$ in dark-energy physics [38]. Hence, $\mathcal{T}_{\mathrm{A}}$ does not respect the third law of thermodynamics. Moreover, one has $\mathcal{T}_{\mathrm{A}}=0$ at $w_{m}=1 / 3$ and thus $\mathcal{T}_{\mathrm{A}} d S_{\mathrm{A}}=0$; following Eq.(41), this can be verified by

$$
\begin{aligned}
A_{\mathrm{A}} \psi & =-A_{\mathrm{A}} H \Upsilon_{\mathrm{A}}\left(1+w_{m}\right) \rho_{m} d t+\frac{1}{2} A_{\mathrm{A}}\left(1+w_{m}\right) \rho_{m} d \Upsilon_{\mathrm{A}} \\
& =A_{\mathrm{A}} \rho_{m}\left(1+w_{m}\right)\left(\frac{1}{2} \dot{\Upsilon}_{\mathrm{A}}-H \Upsilon_{\mathrm{A}}\right) d t \\
& =\frac{9}{8 G} H \Upsilon_{\mathrm{A}}\left(1+w_{m}\right)\left(w_{m}-\frac{1}{3}\right) d t
\end{aligned}
$$

(2) The condition $w_{m}<1 / 3$ for the validity of $\mathcal{T}_{\mathrm{A}}^{(+)}$is too restrictive and unnatural, because $w_{m}=1 / 3$ serves as the EoS of radiation and $(1 \geq) w_{m}>1 / 3$ represents all highly relativistic energy components. For example, it is well known that a canonical and homogeneous scalar field $\phi(t)$ in the FRW Universe has the EoS (e.g. [2])

$$
w_{m}^{(\phi)}=\frac{P_{\phi}}{\rho_{\phi}}=\frac{\frac{1}{2} \dot{\phi}^{2}-V(\phi)}{\frac{1}{2} \dot{\phi}^{2}+V(\phi)} .
$$

$w_{m}^{(\phi)}$ can fall into the domain $1 / 3 \leq w_{m}^{(\phi)} \leq 1$ when the dynamical term $\frac{1}{2} \dot{\phi}^{2}$ dominates over the potential $V(\phi)$, and we donot see any physical reason to a priori rule out this kind of fast-rolling scalar field.

(3) The equality $\mathcal{T}_{\mathrm{A}} d S_{\mathrm{A}}=A_{\mathrm{A}}\left(\psi_{t}+\psi_{\Upsilon}\right)=-\mathcal{T}_{\mathrm{A}}^{(+)} d S_{\mathrm{A}}$ implies that $\mathcal{T}_{\mathrm{A}} d S_{\mathrm{A}}$ and $\mathcal{T}_{\mathrm{A}}^{(+)} d S_{\mathrm{A}}$ need to be balanced by $d t$ and also the $d \Upsilon_{\mathrm{A}}$ component of $\psi$, and thus the other $d \Upsilon_{\mathrm{A}}$ component from $W d V_{\mathrm{A}}=W A_{\mathrm{A}} d \Upsilon_{\mathrm{A}}$ should be nonvanishing as well. Hence, $\mathcal{T}_{\mathrm{A}} d S_{\mathrm{A}}$ and $\mathcal{T}_{\mathrm{A}}^{(+)} d S_{\mathrm{A}}$ always live together with $W d V_{\mathrm{A}}$ to form the total energy differential Eq.(43) rather than some Clausius-type equation $\delta \widetilde{Q}=\mathcal{T}_{\mathrm{A}}^{(+)} d S_{\mathrm{A}}=-\mathcal{T}_{\mathrm{A}} d S_{\mathrm{A}}=-A_{\mathrm{A}} \psi$, and there exists no isochoric process $(d \Upsilon=0)$ for $\left\{\mathcal{T}_{\mathrm{A}}\right.$, $\left.\mathcal{T}_{\mathrm{A}}^{(+)}\right\}$.

(4) The "highly relativistic limit" $w_{m}=1 / 3$ is more than the divide for negative, zero or positive Hayward temperature $\mathcal{T}_{\mathrm{A}}$; it is also the exact divide for the induced metric of the apparent horizon to be spacelike, null or timelike, as discussed before in Sec. II.3. That is to say, the sign of the temperature synchronizes with the signature of the horizon metric. However, there are no such behaviors for analogies in black-hole physics: for example, a slowly-evolving quasilocal black-hole horizon $[41,50]$ can be either spacelike, null or timelike, but the horizon temperature is always positive definite regardless of the horizon signature.

(5) Unlike the Cai-Kim temperature $T_{\mathrm{A}}$, the Hayward $\left\{\mathcal{T}_{\mathrm{A}}\right.$, $\left.\mathcal{T}_{\mathrm{A}}^{(+)}\right\}$used for the problem "from gravitational equations to thermodynamic relations for the Universe" do not work for the problem "from thermodynamic relations to gravitational equations". That is to say, $\left\{\mathcal{T}_{\mathrm{A}}\right.$, $\left.\mathcal{T}_{\mathrm{A}}^{(+)}\right\}$break the symmetry between the formulations of these two mutually inverse problems.

On the other hand, the Cai-Kim temperature $T_{\mathrm{A}}=$ $1 /\left(2 \pi \Upsilon_{\mathrm{A}}\right)$ is positive definite throughout the history of the 
Universe, it provides symmetric formulations of the conjugate problems "gravity to thermodynamics" and "thermodynamics to gravity", and it is the Hawking-like temperature measured by a Kodama observer for the matter tunneling into the untrapped interior $\Upsilon<\Upsilon_{\mathrm{A}}$ from the antitrapped exterior $\Upsilon>\Upsilon_{\mathrm{A}}$ [47]. In fact, besides the assumption Eq.(39) for the approximation $\mathcal{T}_{\mathrm{A}}^{(+)} \approx T_{\mathrm{A}}$ in Eq.(40), there have been efforts to redefine the dynamical surface gravity in place of Eq.(35) for the dynamical apparent horizon $\Upsilon_{\mathrm{A}}$; for example, inspired by the thermodynamics of dynamical black-hole horizons [42], the inaffinity $\kappa:=-\frac{1}{2} \partial_{\Upsilon} \Xi$ with $\Xi:=h^{\alpha \beta} \partial_{\alpha} \Upsilon \partial_{\beta} \Upsilon \equiv 1-\Upsilon^{2} / \Upsilon_{\mathrm{A}}^{2}$ has been employed for the FRW Universe in [48], with which the Cai-Kim temperature satisfies $T_{\mathrm{A}}=\frac{\kappa}{2 \pi}$ at the horizon $\Upsilon=$ $\Upsilon_{\mathrm{A}}$ and thus absorbs the Hayward temperature $\mathcal{T}_{\mathrm{A}}=\kappa /(2 \pi)$.

With these considerations, we adopt the Cai-Kim $T_{\mathrm{A}}$ for the absolute temperature of the cosmological apparent horizon. This way, we believe that the temperature confusion is solved as the Cai-Kim $T_{\mathrm{A}}$ is favored.

Furthermore, imagine a contracting Universe with $\dot{a}<0$ and $H<0$, and one would have a marginally outer trapped apparent horizon with $\theta_{(\ell)}=0$ and $\theta_{(n)}=2 H<0$ at $\Upsilon=\Upsilon_{\mathrm{A}}$. Hence, whether $\Upsilon=\Upsilon_{\mathrm{A}}$ is outer or inner trapped only relies on the Hubble parameter to be negative or positive. In Sec. II.3 we have seen that the induced-metric signature of $\Upsilon_{\mathrm{A}}$ is independent of $H$, and neither will the Hayward $\left\{\mathcal{T}_{\mathrm{A}}\right.$, $\left.\mathcal{T}_{\mathrm{A}}^{(+)}\right\}$. Also, Eqs.(52) and (53) clearly show that, the equality $-T_{\mathrm{A}} d S_{\mathrm{A}}=A_{\mathrm{A}} \psi_{t}=d E_{\mathrm{A}}$ of the Cai-Kim $T_{\mathrm{A}}$ validates for either $H>0$ or $H<0$. Hence, we further conclude that:

Corollary 1 Neither the sign of the Hayward nor the CaiKim temperature is related to the inner or outer trappedness of the cosmological apparent horizon.

\section{IV.4. A quick note on the QCD ghost dark energy}

Among the various types of quantum chromodynamics (QCD) ghost dark energy in existent literature, the following version was introduced in [51] and further discussed in [52],

$$
\rho_{\Lambda}^{(\mathrm{QCD})}=\alpha \Upsilon_{\mathrm{A}}^{-1}\left(1-\frac{\dot{\Upsilon}_{\mathrm{A}}}{2 H \Upsilon_{\mathrm{A}}}\right),
$$

where $\alpha$ is a positive constant with the dimension of [energy] $]^{3}$. It is based on the idea that the vacuum energy density is proportional to the temperature of the apparent horizon $\Upsilon_{\mathrm{A}}$, which was chosen as the Hayward $\left\{\mathcal{T}_{\mathrm{A}}, \mathcal{T}_{\mathrm{A}}^{(+)}\right\}$in [51]. Following the discussion just above, we can see that Eq.(51) turns out to be problematic because $\rho_{\Lambda}^{(\mathrm{QCD})}$ is not positive definite, with $\rho_{\Lambda}^{(\mathrm{QCD})} \leq 0$ when the Universe is dominated by superrelativistic matter $1 / 3 \leq w_{m}(\leq 1)$. In fact, more viable forms of the QCD ghost dark energy can be found in e.g. [53].

\section{THE (GENERALIZED) SECOND LAWS OF THERMODYNAMICS}

Having studied the differential forms of the energy conservation and heat transfer and distinguished the temperature of of the apparent horizon, we will proceed to investigate the entropy evolution for the Universe.

\section{V.1. Positive heat out thermodynamic sign convention}

As a corner stone for our formulation of the second laws and solution to the entropy confusion, we will match the thermodynamic sign convention encoded in the Cai-Kim Clausius equation $T_{\mathrm{A}} d S_{\mathrm{A}}=\delta Q_{\mathrm{A}}=-A_{\mathrm{A}} \psi_{t}$. Following Secs. III.1 and III.2, we first check whether the heat flow element $\delta Q_{\mathrm{A}}$ and the isochoric energy differential $d E_{\mathrm{A} 0}=d\left(\rho_{m} V_{\mathrm{A} 0}\right)$ take positive or negative values. $\delta Q_{\mathrm{A}}$ will be calculated by $T_{\mathrm{A}} d S_{\mathrm{A}}$, while $d E_{\mathrm{A}}$ is to be evaluated independently via $A_{\mathrm{A}} \psi_{t}=$ $-A_{\mathrm{A}}\left(1+w_{m}\right) \rho_{m} H \Upsilon_{\mathrm{A}} d t$. Hence, in the isochoric process for an instantaneous apparent horizon $\Upsilon_{\mathrm{A} 0}$,

$$
\begin{gathered}
T_{\mathrm{A}} d S_{\mathrm{A}}=\frac{\dot{\Upsilon}_{\mathrm{A}}}{G} d t=\frac{3}{2 G} H \Upsilon_{\mathrm{A}}\left(1+w_{m}\right) d t \\
\left.d E\right|_{\Upsilon_{\mathrm{A} 0}}=-A_{\mathrm{A}} \rho_{m}\left(1+w_{m}\right) H \Upsilon_{\mathrm{A}} d t=-\frac{3}{2 G} H \Upsilon_{\mathrm{A}}\left(1+w_{m}\right) d t
\end{gathered}
$$

where Eqs. (10) and (11) have been used to replace $A_{\mathrm{A}} \rho_{m}$ and $\dot{\Upsilon}_{\mathrm{A}}$, respectively. For an expanding Universe $(H>0)$, this clearly shows that:

(1) If the Universe is dominated by ordinary matter or quintessence, $-1<w_{m}(\leq 1)$, the internal energy is decreasing $d E_{\mathrm{A}}=A_{\mathrm{A}} \boldsymbol{\psi}_{t}<0$, with a positive Hubble energy flow $\delta Q_{\mathrm{A}}=T_{\mathrm{A}} d S_{\mathrm{A}}>0$ going outside to the surroundings;

(2) Under the dominance of the cosmological constant, $w_{m}=-1$ and $\left\{\rho_{m}, \Upsilon_{\mathrm{A}}, T_{\mathrm{A}}, S_{\mathrm{A}}\right\}=$ constant; the internal energy is unchanging, $d E_{\mathrm{A}}=A_{\mathrm{A}} \psi_{t}=0$ and $\delta Q_{\mathrm{A}}=T_{\mathrm{A}} d S_{\mathrm{A}}=0$;

(3) When the Universe enters the phantom-dominated state, $w_{m}<-1$, the internal energy increases $d E_{\mathrm{A}}=A_{\mathrm{A}} \psi_{t}>$ 0 while $\delta Q_{\mathrm{A}}=T_{\mathrm{A}} d S_{\mathrm{A}}<0$.

Hence, based on the intuitive behaviors at the domain $-1<$ $w_{m}(\leq 1)$ for nonexotic matter, we set up the positive heat out thermodynamic sign convention for the right hand side of $d E_{\mathrm{A}}=-\delta Q_{\mathrm{A}}$. That is to say, heat emitted by the system takes positive values $\left(\delta Q_{\mathrm{A}}=\delta Q_{\mathrm{A}}^{\text {out }}>0\right)$, while heat absorbed by the system takes negative values. Obviously, this setup is totally consistent with the situations of $w_{m} \leq-1$. Also, because of the counterintuitive behaviors under phantom dominance, one should not take it for granted that, for a spatially expanding Universe the cosmic fluid would always flow out of the isochoric volume $V\left(\Upsilon=\Upsilon_{0}\right)$ with $d E=V_{\mathrm{A} 0} d \rho_{m}<0$. 


\section{V.2. Positive heat out Gibbs equation}

In existent papers, the cosmic entropy is generally studied independently of the first laws, and the entropy $\widehat{S}_{m}$ of the cosmic energy-matter content (with temperature $T_{m}$ ) is always determined by the traditional Gibbs equation $d E=$ $T_{m} d \widehat{S}_{m}-P_{m} d V$ (e.g. [14]-[24]). This way, $\dot{\widehat{S}}_{m}$ departs dramatically from the expected non-decreasing behaviors, so people turn to the generalized version of the second law for help, which works with the sum of $\widehat{S}_{m}$ and the geometric entropy of the cosmological apparent or event horizons.

This popular treatment is very problematic. In fact, the equation $d E_{m}=T_{m} d \widehat{S}_{m}-P_{m} d V$ encodes the "positive heat in, positive work out" convention for the physical entropy $\widehat{S}_{m}$ and the heat transfer $T_{m} d \widehat{S}_{m}$. However, as extensively discussed just above, the geometric Bekenstein-Hawking entropy $S_{\mathrm{A}}=A_{\mathrm{A}} / 4 G$ for the cosmological apparent horizon is compatible with the positive-heat-out convention. One cannot add the traditional positive-heat-in $\widehat{S}_{m}$ with the positive-heatout $S_{A}$, and this conflict ${ }^{1}$ leads us to adjust the Gibbs equation into

$$
d E_{m}=-T_{m} d S_{m}-P_{m} d V
$$

where $S_{m}$ is defined in the positive-heat-out convention favored by the Universe for consistency with the holographicstyle gravitational equations (8), (9) and (12). This way, one can feel free and safe to superpose or compare the matter entropy $S_{m}$ and the horizon entropy $\left\{S_{\mathrm{A}}\right.$, etc. $\}$, and even more pleasantly, it turns out that this $S_{m}$ is very well behaved.

Moreover, note that although the Gibbs equation is usually derived from a reversible process in a closed system ("controlled mass"), Eq.(54) actually applies to either reversible or irreversible processes, and either closed or open systems, because it only contains state quantities which are independent of thermodynamic processes.

For the energy $E=M=\rho_{m} V$ in an arbitrary volume $V=$ $\frac{4}{3} \pi \Upsilon^{3}=\frac{1}{3} A \Upsilon$, Eq. (54) yields $T_{m} d S_{m}=-d\left(\rho_{m} V\right)-P_{m} d V=$ $-V d \rho_{m}-\left(\rho_{m}+P_{m}\right) d V$, and thus

$$
\begin{aligned}
T_{m} d S_{m} & =3 H\left(\rho_{m}+P_{m}\right) V d t-\left(\rho_{m}+P_{m}\right) A d \Upsilon \\
& =\rho_{m} A\left(1+w_{m}\right)(H \Upsilon d t-d \Upsilon),
\end{aligned}
$$

where the continuity equation (16) has been used. Based on Eq.(55), we can analyze the entropy evolution $\dot{S}_{m}$ for the matter inside some special radii such as the apparent and event horizons. Note that these regions are generally open thermodynamic systems with the Hubble energy flow crossing the apparent and possibly the event horizons, so one should not $a$ priori anticipate $\dot{S}_{m} \geq 0$; instead, we will look for the circumstances where $\dot{S}_{m} \geq 0$ conditionally holds.

\footnotetext{
${ }^{1}$ Note that there is no such conflict for black holes, because both the blackhole horizon entropy and the matter entropy are defined in the positiveheat-in convention.
}

\section{V.3. The second law for the interior of the apparent horizon}

For the matter inside the apparent horizon $\Upsilon=\Upsilon_{\mathrm{A}}(t)$, Eq.(55) along with the holographic-style dynamical equations (10) and (11) yield

$$
\begin{aligned}
T_{m} d S_{m}^{(\mathrm{A})} & =\rho_{m} A_{\mathrm{A}}\left(1+w_{m}\right)\left(H \Upsilon_{\mathrm{A}}-\dot{\Upsilon}_{\mathrm{A}}\right) d t \\
& =\frac{3}{2 G}\left(1+w_{m}\right) H \Upsilon_{\mathrm{A}}\left(1-\frac{3}{2}\left(1+w_{m}\right)\right) d t \\
& =-\frac{9}{4 G} H \Upsilon_{\mathrm{A}}\left(w_{m}+1\right)\left(w_{m}+\frac{1}{3}\right) d t
\end{aligned}
$$

Obviously the second law of thermodynamics $\dot{S}_{m}^{(\mathrm{A})} \geq 0$ holds for $-1 \leq w_{m} \leq-1 / 3$. Moreover, recall that the spatial expansion of the generic FRW Unverse satisfies

$$
\frac{\ddot{a}}{a}=-\frac{4 \pi G}{3}\left(1+3 w_{m}\right) \rho_{m},
$$

with $\ddot{a}>0$ for $w_{m}<-1 / 3$. Hence, within GR and the $\Lambda$ CDM model, we have:

Theorem 1 The physical entropy $S_{m}^{(A)}$ inside the cosmological apparent horizon satisfies $\dot{S}_{m}^{(A)} \equiv 0$ when $w_{m}=-1 / 3$ or under the dominance of the cosmological constant $w_{m}=-1$, while $\dot{S}_{m}^{(A)}>0$ for the stage of accelerated expansion $(\ddot{a}>0)$ dominated by quintessence $-1<w_{m}<-1 / 3$.

\section{V.4. The second law for the interior of the event and particle horizons}

Consider the future-pointed cosmological event horizon $\Upsilon_{\mathrm{E}}:=a \int_{t}^{\infty} a^{-1} d \hat{t}$ which measures the distance that light signals will travel over the entire future history from $\hat{t}_{0}=t . \Upsilon_{\mathrm{E}}$ satisfies

$$
\dot{\Upsilon}_{\mathrm{E}}=H \Upsilon_{\mathrm{E}}-1
$$

so for the cosmic fluid inside $\Upsilon_{E}$, Eq.(55) leads to

$$
\begin{aligned}
T_{m} d S_{m}^{(\mathrm{E})} & =\rho_{m} A_{\mathrm{E}}\left(1+w_{m}\right)\left(H \Upsilon_{\mathrm{E}}-\dot{\Upsilon}_{\mathrm{E}}\right) d t \\
& =\rho_{m} A_{\mathrm{E}}\left(1+w_{m}\right) d t .
\end{aligned}
$$

Hence, we are very happy to see that:

Theorem 2 The physical entropy $S_{m}^{(E)}$ inside the cosmological event horizon satisfies $\dot{S}_{m}^{(E)} \equiv 0$ if the Universe is dominated by the cosmological constant $w_{m}=-1$, while $\dot{S}_{m}^{(E)}>0$ for all nonexotic matter $-1<w_{m}(\leq 1)$ above the phantom divide.

The importance of this result can be best seen for a closed $(k=1)$ Universe, when the event horizon $\Upsilon_{\mathrm{E}}$ has a finite radius and bounds the entire spacetime. Then the physical entropy of the whole Universe is nondecreasing as long as the dominant energy condition holds $-1 \leq w_{m}(\leq 1)$.

Similarly for the past particle horizon $\Upsilon_{\mathrm{P}}:=a \int_{0}^{t} a^{-1} d \hat{t}$ (e.g. 
$[26,43,54])$, which supplements the event horizon $\Upsilon_{E}$ and measures the distance that light has already traveled from the beginning of time (or equivalently the most distant objects one could currently observe), it satisfies $\dot{\Upsilon}_{\mathrm{P}}=H \Upsilon_{\mathrm{P}}+1$ and thus Eq.(55) yields

$$
\begin{aligned}
T_{m} d S_{m}^{(\mathrm{P})} & =\rho_{m} A_{\mathrm{P}}\left(1+w_{m}\right)\left(H \Upsilon_{\mathrm{P}}-\dot{\Upsilon}_{\mathrm{P}}\right) d t \\
& =-\rho_{m} A_{\mathrm{P}}\left(1+w_{m}\right) d t
\end{aligned}
$$

Besides $\dot{S}_{m}^{(\mathrm{P})} \equiv 0$ for $w_{m}=-1, \dot{S}_{m}^{(\mathrm{P})}<0$ always holds at the domain $-1<w_{m}(\leq 1)$, which means that the physical entropy is always decreasing when we trace back to the earlier age for the younger Universe that has a larger particle horizon radius $\Upsilon_{\mathrm{P}}$ or horizon area $A_{\mathrm{P}}$.

Note that with the traditional Gibbs equation $d E_{m}=$ $T_{m} d \widehat{S}_{m}-P_{m} d V$ where $\widehat{S}_{m}$ is defined in the positive-heat-in convention, for the interiors of the future $\Upsilon_{\mathrm{E}}$ and the past $\Upsilon_{\mathrm{P}}$ one would always obtain

$$
\begin{aligned}
& T_{m} d \widehat{S}_{m}^{(\mathrm{E})}=d E_{m}^{(\mathrm{E})}+P_{m} d V_{\mathrm{E}}=-\rho_{m} A_{\mathrm{E}}\left(1+w_{m}\right) d t \\
& T_{m} d \widehat{S}_{m}^{(\mathrm{P})}=d E_{m}^{(\mathrm{P})}+P_{m} d V_{\mathrm{P}}=\rho_{m} A_{\mathrm{P}}\left(1+w_{m}\right) d t
\end{aligned}
$$

It would imply that in the future $\dot{\vec{S}}_{m}^{(\mathrm{E})}>0$ would never be realized and a younger Universe (larger $A_{\mathrm{P}}$ ) would however carry a larger internal entropy $\widehat{S}_{m}^{(\mathrm{P})}$, unless the Universe were in an exotically phantom-dominated $\left(w_{m}<-1\right)$ state in her history. We believe that Eqs. $(59,60)$ provide a more reasonable description for the cosmic entropy evolution than Eq.(61), regard this result as a support to the positive-heat-out Gibbs equation (54), and argue that Eqs.(54), (59) and (60) have solved the cosmological entropy confusion caused by Eq.(61) in traditional studies.

\section{V.5. GSL for the apparent-horizon system}

Historically, to rescue the disastrous result of the traditional Eq.(61), the generalized second law (GSL) for the thermodynamics of the Universe was developed, which adds up the geometrically defined entropy of the cosmological boundaries (mainly $S_{\mathrm{A}}, S_{\mathrm{E}}$ ) to the physical entropy of the matter-energy content $S_{m}$, aiming to make the total entropy nondecreasing under certain conditions. This idea is inspired by the GSL of black-hole thermodynamics [49], for which Bekenstein postulated that the black-hole horizon entropy plus the external matter entropy never decrease (for a thermodynamic closed system).

Eq.(59) clearly indicates that the second law $\dot{S}_{m} \geq 0$ is well respected in our formulation, but for completeness we will still re-investigate the GSLs. For the simple open system consisting of the cosmological apparent horizon $\Upsilon_{\mathrm{A}}$ and its inte- rior, Eqs.(29) and (56) yields

$$
\begin{aligned}
\dot{S}_{m}^{(\mathrm{A})}+\dot{S}_{\mathrm{A}}= & -\frac{1}{T_{m}} \frac{9}{4 G} H \Upsilon_{\mathrm{A}}\left(w_{m}+1\right)\left(w_{m}+\frac{1}{3}\right)+\frac{2 \pi \Upsilon_{\mathrm{A}} \dot{\Upsilon}_{\mathrm{A}}}{G} \\
= & -\frac{1}{T_{m}} \frac{9}{4 G} H \Upsilon_{\mathrm{A}}\left(w_{m}+1\right)\left(w_{m}+\frac{1}{3}\right) \\
& +\frac{1}{T_{\mathrm{A}}} \frac{3}{2 G} H \Upsilon_{\mathrm{A}}\left(w_{m}+1\right) .
\end{aligned}
$$

In existing papers it is generally assumed that the apparent horizon would be in thermal equilibrium with the matter content and thus $T_{\mathrm{A}}=T_{m}[16,19-22,24,25]$, or occasionally less restrictively $T_{m}=b T_{\mathrm{A}}(b=$ constant $)[17,18]$. However, such assumptions are essentially mathematical tricks to simplify Eq.(62), while physically they are too problematic, so we directly move ahead from Eq.(62) without any artificial speculations relating $T_{\mathrm{A}}$ and $T_{m}$.

The GSL $\dot{S}_{m}^{(\mathrm{A})}+\dot{S}_{\mathrm{A}} \geq 0$ could hold when $\frac{1}{T_{\mathrm{A}}} \frac{3}{2 G} H \Upsilon_{\mathrm{A}}\left(w_{m}+\right.$ 1) $\geq \frac{1}{T_{m}} \frac{9}{4 G} H \Upsilon_{\mathrm{A}}\left(w_{m}+1\right)\left(w_{m}+\frac{1}{3}\right)$, and with $\left\{H, \Upsilon_{\mathrm{A}}, T_{\mathrm{A}}, T_{m}\right\}>0$ it leads to

$$
\left(w_{m}+1\right)\left(\frac{T_{m}}{T_{\mathrm{A}}}-\frac{3}{2}\left(w_{m}+\frac{1}{3}\right)\right) \geq 0,
$$

or equivalently $\left(w_{m}+1\right)\left(T_{m}-\frac{3}{2}\left(w_{m}+\frac{1}{3}\right) T_{\mathrm{A}}\right) \geq 0$. Hence, for the apparent-horizon system the GSL trivially validates with $\dot{S}_{m}+$ $\dot{S}_{\mathrm{A}} \equiv 0$ under the dominance of the cosmological constant $w_{m}=-1$, and:

(1) For $-1<w_{m}<-1 / 3$ which corresponds to an accelerated Universe dominated by quintessence, $\dot{S}_{m}+\dot{S}_{\mathrm{A}}>0$ always holds, because $T_{m} / T_{\mathrm{A}}>0$ and $\frac{3}{2}\left(w_{m}+\frac{1}{3}\right)<0$ [or because both $\dot{S}_{m}>0$ and $\dot{S}_{\mathrm{A}}>0$ ];

(2) For $-1 / 3 \leq w_{m}(\leq 1)$ which corresponds to ordinarymatter dominance respecting the strong energy condition $\rho_{m}+3 P_{m} \geq 0$ [40], the GSL $\dot{S}_{m}+\dot{S}_{\mathrm{A}} \geq 0$ conditionally holds when

$$
\frac{T_{m}}{T_{\mathrm{A}}} \geq \frac{3}{2}\left(w_{m}+\frac{1}{3}\right) ;
$$

(3) For the phantom domain $w_{m}<-1$, the GSL never validates because it requires $T_{m} / T_{\mathrm{A}} \leq \frac{3}{2}\left(w_{m}+\frac{1}{3}\right)<0$ which violates the the third law of thermodynamics.

\section{V.6. GSL for the event-horizon system}

Now consider the system made up of the cosmological event horizon and its interior. Unlike the apparent horizon, the entropy $S_{\mathrm{E}}$ and temperature $T_{\mathrm{E}}$ of the event horizon $\Upsilon_{\mathrm{E}}$ are unknown yet; one should not take it for granted that $\Upsilon_{E}$ would still carry the Bekenstein-Hawking entropy $S_{\mathrm{E}}=A_{\mathrm{E}} / 4 G$ and further assume a Cai-Kim temperature $T_{\mathrm{E}}=1 /\left(2 \pi \Upsilon_{\mathrm{E}}\right)$ to it.

Considering that $S_{\mathrm{E}}$ would reflect the amount of Hubbleflow energy crossing an instantaneous event horizon $\Upsilon_{\mathrm{E}}=$ $\Upsilon_{\mathrm{E} 0}$, it is still safe to make use of the unified first law Eq.(22) 
and thus

$$
T_{\mathrm{E}} d S_{\mathrm{E}}=\delta Q_{\mathrm{E}}=-\left.d E\right|_{\Upsilon_{\mathrm{E} 0}}=A_{\mathrm{E}}\left(1+w_{m}\right) \rho_{m} H \Upsilon_{\mathrm{E}} d t .
$$

Hence for the event horizon system we have

$$
\begin{aligned}
\dot{S}_{m}^{(\mathrm{E})}+\dot{S}_{\mathrm{E}} & =\frac{1}{T_{m}}\left(1+w_{m}\right) \rho_{m} A_{\mathrm{E}}+\frac{1}{T_{\mathrm{E}}} A_{\mathrm{E}}\left(1+w_{m}\right) \rho_{m} H \Upsilon_{\mathrm{E}} \\
& =\rho_{m} A_{\mathrm{E}}\left(1+w_{m}\right)\left(\frac{1}{T_{m}}+\frac{1}{T_{\mathrm{E}}} \frac{\Upsilon_{\mathrm{E}}}{\Upsilon_{\mathrm{H}}}\right),
\end{aligned}
$$

where $\Upsilon_{H}:=1 / H$ refers to the radius of the Hubble horizon $[30,43]$, an auxiliary scale where the recession speed would reach that of light ( $c=1$ in our units) by Hubble's law, and it is more instructive to write $H$ as $1 / \Upsilon_{\mathrm{H}}$ when compared with $\Upsilon_{\mathrm{E}}$ and $\Upsilon_{\mathrm{A}}$. Since $\left\{T_{\mathrm{A}}, T_{\mathrm{E}}, H, \Upsilon_{\mathrm{E}}\right\}>0$, we pleasantly conclude from Eq.(66) without any unnatural assumption on $\left\{T_{m}, T_{\mathrm{E}}\right\}$ that:

Theorem 3 The GSL $\dot{S}_{m}^{(E)}+\dot{S}_{E} \geq 0$ for the event horizon system always holds for an expanding Universe dominated by nonexotic matter $-1 \leq w_{m}(\leq 1)$.

Note that Mazumder and Chakraborty have discussed GSLs for the event-horizon system in various dark-energy (and modified-gravity) models in $[55,56]$, where $S_{\mathrm{E}}$ is calculated by the unified first law and the importance of $w_{m}$ is fully realized, although it is the weak rather than the dominant energy condition that is emphasized therein and the possibility of a Bekenstein-Hawking entropy for $\Upsilon_{\mathrm{E}}$ is not analyzed.

So far we have seen that though the apparent horizon $\Upsilon_{\mathrm{A}}$ is more compatible with the unified first law and the Clausius equation, the second law is better respected by the cosmic fluid inside the event horizon $\Upsilon_{E}$ - this is because $\Upsilon_{E}$ better captures the philosophical concept of "the whole Universe". For both horizons $\Upsilon_{\mathrm{A}}$ and $\Upsilon_{\mathrm{E}}$, the second law is better formulated than the GSL. Moreover, from the standpoint of the second laws and the GSLs, the phantom $\left(w_{m}<-1\right)$ dark energy is definitely less favored than the cosmological constant $\left(w_{m}=-1\right)$ and the quintessence $\left(-1<w_{m}<-1 / 3\right)$.

\section{V.7. Bekenstein-Hawking entropy and Cai-Kim temperature for the event horizon?}

The entropy of the event horizon $\Upsilon_{\mathrm{E}}$ has just been calculated from the unified fist law. Now let's return to the question: Can the Bekenstein-Hawking entropy and/or the CaiKim temperature be applied to $\Upsilon_{\mathrm{E}}$ ? With the assumption $S_{\mathrm{E}}=A_{\mathrm{E}} / 4 G$, Eq.(65) yields

$T_{\mathrm{E}} \frac{2 \pi \Upsilon_{\mathrm{E}} \dot{\Upsilon}_{\mathrm{E}}}{G}=T_{\mathrm{E}} \frac{2 \pi \Upsilon_{\mathrm{E}}\left(H \Upsilon_{\mathrm{E}}-1\right)}{G}=A_{\mathrm{E}}\left(1+w_{m}\right) \rho_{m} H \Upsilon_{\mathrm{E}}$

which further leads to

$$
\left(\Upsilon_{\mathrm{E}}-\Upsilon_{\mathrm{H}}\right) T_{\mathrm{E}}=\frac{G}{2 \pi} \rho_{m} A_{\mathrm{E}}\left(1+w_{m}\right) .
$$

An expanding FRW Universe always satisfies $\Upsilon_{\mathrm{E}} \geq \Upsilon_{\mathrm{H}}$, so the third law of thermodynamics $T_{\mathrm{E}}>0$ requires $-1 \leq w_{m}(\leq 1)$; also, $\Upsilon_{\mathrm{E}}=\Upsilon_{\mathrm{H}}$ when $w_{m}=-1$ and $T_{\mathrm{E}}$ becomes unspecifiable from Eq.(68). Moreover, if $\Upsilon_{\mathrm{E}}=\Upsilon_{\mathrm{H}}$, then $a \int_{t}^{\infty} a^{-1} d \hat{t}=\frac{a}{\dot{a}}$, thus

$$
\dot{a} \int_{t}^{\infty} a^{-1} d \hat{t}=1 \quad \Rightarrow \quad \frac{\ddot{a}}{\dot{a}}-\frac{\dot{a}}{a}=0 \quad \Rightarrow \quad a \ddot{a}=\dot{a}^{2},
$$

where we have taken the time derivative of the left-most integral expression. In the meantime, when $w_{m}=-1$ we have

$$
\frac{\dot{a}^{2}+k}{a^{2}}=\frac{8}{3} \pi G \rho_{m} \quad, \quad \frac{\ddot{a}}{a}=\frac{8}{3} \pi G \rho_{m} \quad \Rightarrow a \ddot{a}=\dot{a}^{2}+k .
$$

Comparison of Eqs.(69) and (70) shows that in addition to $w_{m}=-1, \Upsilon_{\mathrm{E}}=\Upsilon_{\mathrm{H}}$ also requires $k=0$; note that in case of the flat Universe, the apparent and the Hubble horizons coincide, $\Upsilon_{\mathrm{A}}=\Upsilon_{\mathrm{H}}$, so $T_{\mathrm{E}}=T_{\mathrm{A}}$ which remedies the failure of Eq.(68) at $w_{m}=-1$. Hence,

Corollary 2 The validation of a Bekenstein-Hawking entropy on the cosmological event horizon requires that (i) the scale factor a(t) satisfies the constraint Eq.(68), (ii) the dominant energy condition always holds, (iii) the event and Hubble horizons would coincide and the spatial curvature vanishes under the dominance of the cosmological constant.

If one further assumes a Cai-Kim-like $T_{\mathrm{E}}=1 /\left(2 \pi \Upsilon_{\mathrm{E}}\right)$ for the event horizon, Eq.(68) would tell us that

$$
G \rho_{m} A_{\mathrm{E}}\left(1+w_{m}\right)+\frac{\Upsilon_{\mathrm{H}}}{\Upsilon_{\mathrm{E}}}=1 .
$$

Does this constraint always hold? Since $\Upsilon_{E} \geq \Upsilon_{A}$, thus $\rho_{m} A_{\mathrm{E}} \geq \rho_{m} A_{\mathrm{A}}=\frac{3}{2 G}$, with which Eq.(71) yields

$$
\frac{3}{2}\left(1+w_{m}\right)+\frac{\Upsilon_{\mathrm{H}}}{\Upsilon_{\mathrm{E}}} \leq 1 .
$$

This result can be rearranged into

$$
w_{m} \leq-\frac{1}{3}-\frac{\Upsilon_{\mathrm{H}}}{\Upsilon_{\mathrm{E}}}<-\frac{1}{3},
$$

which, together with the requirement $-1 \leq w_{m}(\leq 1)$ from Eq.(68) for a generic positive $T_{\mathrm{E}}$, give rise to the condition $-1 \leq w_{m}<-1 / 3$. Hence,

Corollary 3 In addition to a Bekenstein-Hawking entropy, the validation of a Cai-Kim temperature on the cosmological event horizon further requires the scale factor to satisfy Eq.(71), and restricts the FRW Universe to be dominated by the cosmological constant $w_{m}=-1$ or quintessence $-1<w_{m}<-1 / 3$.

Similar conditions hold for the past particle horizon as well. [54] has derived the GSL inequalities for the Hubble-, apparent-, particle- and event-horizon systems with the logamediate and intermediate scale factors by both the first law 
and the Bekenstein-Hawking formula, in which one could clearly observe that these two methods yield different results in the case of the event (and particle, Hubble) horizons.

Based on these considerations we argue that for consistency with the cosmic gravitational dynamics, the geometrically defined $A / 4 G$ only unconditionally holds on the apparent horizon $\Upsilon_{\mathrm{A}}$, which does not support the belief that the BekensteinHawking entropy could validate for all horizons in GR (e.g. $[14,43])$.

\section{GRAVITATIONAL THERMODYNAMICS IN ORDINARY MODIFIED GRAVITIES}

For the $\Lambda$ CDM Universe within GR, we have re-studied the first and second laws of thermodynamics by requiring the consistency with the holographic-style dynamical equations (8), (9) and (11), which provides possible solutions to the long-standing temperature and entropy confusions. Following the clarification of the Cai-Kim temperature and the positive-heat-out sign convention, we will take this opportunity to extend the whole framework of gravitational thermodynamics to modified and alternative theories of relativistic gravity [57, 58]; also, this is partly a continuation of our earlier work in [64] where a unified formulation has been developed to derive the cosmological dynamical equations in modified gravities from (non)equilibrium thermodynamics.

For the generic Lagrangian density $\mathscr{L}_{\text {total }}=$ $\mathscr{L}_{G}\left(R, R_{\mu \nu} R^{\mu \nu}, \mathcal{R}_{i}, \vartheta, \nabla_{\mu} \vartheta \nabla^{\mu} \vartheta, \cdots\right)+16 \pi G \mathscr{L}_{m}$, where $\mathcal{R}_{i}=\mathcal{R}_{i}\left(g_{\alpha \beta}, R_{\mu \alpha \nu \beta}, \nabla_{\gamma} R_{\mu \alpha \nu \beta}, \ldots\right)$ refers to a generic Riemannian invariant beyond the Ricci scalar and $\vartheta$ denotes a scalarial extra degree of freedom unabsorbed by $\mathscr{L}_{m}$, the field equation reads

$$
H_{\mu \nu}=8 \pi G T_{\mu \nu}^{(m)} \text { with } H_{\mu v}:=\frac{1}{\sqrt{-g}} \frac{\delta\left(\sqrt{-g} \mathscr{L}_{G}\right)}{\delta g^{\mu \nu}},
$$

where total-derivative/boundary terms should be removed in the derivation of $H_{\mu \nu}$. In the spirit of reconstructing the effective dark energy [63], Eq.(74) can be intrinsically recast into a compact GR form by isolating the $R_{\mu \nu}$ in $H_{\mu \nu}$ :

$$
\begin{gathered}
G_{\mu \nu} \equiv R_{\mu \nu}-\frac{1}{2} R g_{\mu \nu}=8 \pi G_{\mathrm{eff}} T_{\mu \nu}^{(\mathrm{eff})} \text { with } \\
H_{\mu \nu}=\frac{G}{G_{\mathrm{eff}}} G_{\mu \nu}-8 \pi G T_{\mu \nu}^{(\mathrm{MG})}
\end{gathered}
$$

where $T_{\mu \nu}^{(\mathrm{eff})}=T_{\mu \nu}^{(m)}+T_{\mu \nu}^{(\mathrm{MG})}$, and all terms beyond GR have been packed into $T_{\mu \nu}^{(\mathrm{MG})}$ and $G_{\text {eff. }}$ Here $T_{\mu \nu}^{(\mathrm{MG})}$ collects the modified-gravity nonlinear and higher-order effects, while $G_{\text {eff }}$ denotes the effective gravitational coupling strength which can be directly recognized from the coefficient of the matter tensor $T_{\mu \nu}^{(m)}$ - for example, as will be shown in Sec.VI.8, we have $G_{\text {eff }}=G / f_{R}$ for $f(R), G_{\text {eff }}=G E(\phi) / F(\phi)$ for scalar-tensor-chameleon, $G_{\text {eff }}=G / \phi$ for Brans-Dicke, $G_{\text {eff }}=$ $G /(1+2 a R)$ for quadratic, and $G_{\text {eff }}=G$ for dynamical ChernSimons gravities. Moreover, $T_{\mu \nu}^{(\mathrm{eff})}$ is assumed to be an effec- tive perfect-fluid content,

$$
\begin{aligned}
& T_{v}^{\mu(\mathrm{eff})}=\operatorname{diag}\left[-\rho_{\mathrm{eff}}, P_{\mathrm{eff}}, P_{\mathrm{eff}}, P_{\mathrm{eff}}\right] \\
& \text { with } \quad P_{\mathrm{eff}} / \rho_{\mathrm{eff}}=: w_{\mathrm{eff}},
\end{aligned}
$$

along with $\rho_{\text {eff }}=\rho_{m}+\rho_{(\mathrm{MG})}$ and $P_{\text {eff }}=P_{m}+P_{(\mathrm{MG})}$.

Modified gravities aim to explain the cosmic acceleration without dark-energy components, so in this section we will assume the physical matter to respect the null, weak, strong and dominant energy conditions [40], which yield $\rho_{m}>0$ and $-1 / 3 \leq w_{m} \leq 1$. This way, the quintessence $\left(-1<w_{m}<\right.$ $-1 / 3)$, the cosmological constant $\left(w_{m}=-1\right)$ and the most exotic phantom $\left(w_{m}<-1\right)$ are ruled out.

\section{VI.1. Holographic-style dynamical equations in modified gravities}

Substituting the FRW metric Eq.(1) and the effective cosmic fluid Eq.(76) into the field equation (75), one could obtain the modified Friedmann equations

$$
\begin{gathered}
H^{2}+\frac{k}{a^{2}}=\frac{8 \pi G_{\mathrm{eff}}}{3} \rho_{\mathrm{eff}} \quad \text { and } \\
\dot{H}-\frac{k}{a^{2}}=-4 \pi G_{\mathrm{eff}}\left(1+w_{\mathrm{eff}}\right) \rho_{\mathrm{eff}}=-4 \pi G_{\mathrm{eff}} h_{\mathrm{eff}} \\
\text { or } 2 \dot{H}+3 H^{2}+\frac{k}{a^{2}}=-8 \pi G_{\mathrm{eff}} P_{\mathrm{eff}},
\end{gathered}
$$

where $h_{\mathrm{eff}}:=\left(1+w_{\mathrm{eff}}\right) \rho_{\mathrm{eff}}$ denotes the effective enthalpy density. With Eqs. (4) and (5), substituting the apparent-horizon radius $\Upsilon_{\mathrm{A}}$ and its kinematic time-derivative $\dot{\Upsilon}_{\mathrm{A}}$ into Eq.(77), the Friedmann equations can be rewritten into

$$
\begin{gathered}
\Upsilon_{\mathrm{A}}^{-2}=\frac{8 \pi G_{\text {eff }}}{3} \rho_{\text {eff }} \\
\dot{\Upsilon}_{\mathrm{A}}=4 \pi H \Upsilon_{\mathrm{A}}^{3} G_{\mathrm{eff}}\left(1+w_{\mathrm{eff}}\right) \rho_{\mathrm{eff}} \\
=\frac{3}{2} H \Upsilon_{\mathrm{A}}\left(1+w_{\mathrm{eff}}\right) \\
\Upsilon_{\mathrm{A}}^{-3}\left(\dot{\Upsilon}_{\mathrm{A}}-\frac{3}{2} H \Upsilon_{\mathrm{A}}\right)=4 \pi G_{\text {eff }} H P_{\mathrm{eff}},
\end{gathered}
$$

along with $A_{\mathrm{A}} \rho_{\mathrm{eff}}=\frac{3}{2 G_{\text {eff }}}$. Similar to Eqs.(8)-(12) for $\Lambda \mathrm{CDM}$ of GR, Eqs.(78)-(81) constitute the full set of FRW holographic-style gravitational equations for modified gravities of the form Eq.(75).

\section{VI.2. Unified first law of nonequilibrium thermodynamics}

Following our previous work [64], to geometrically reconstruct the effective total internal energy $E_{\text {eff }}$, one just needs to replace Newton's constant $G$ by $G_{\text {eff }}$ in the standard MisnerSharp or Hawking mass used in Sec. III.1, which yields

$$
E_{\mathrm{eff}}=\frac{1}{2 G_{\mathrm{eff}}} \frac{\Upsilon^{3}}{\Upsilon_{\mathrm{A}}^{2}}
$$


The total derivative of $E_{\mathrm{eff}}=E_{\mathrm{eff}}(t, r)$ along with the holographic-style dynamical equations (78), (79) and (81) yield

$$
\begin{aligned}
d E_{\mathrm{eff}}= & -\frac{1}{G_{\mathrm{eff}}} \frac{\Upsilon^{3}}{\Upsilon_{\mathrm{A}}^{3}}\left(\dot{\Upsilon}_{\mathrm{A}}-\frac{3}{2} H \Upsilon_{\mathrm{A}}\right) d t+\frac{3}{2 G_{\mathrm{eff}}} \frac{\Upsilon^{2}}{\Upsilon_{\mathrm{A}}^{2}} a d r \\
& -\frac{\dot{G}_{\mathrm{eff}}}{2 G_{\mathrm{eff}}^{2}} \frac{\Upsilon^{3}}{\Upsilon_{\mathrm{A}}^{2}} d t \\
= & -A \Upsilon H P_{\mathrm{eff}} d t+A \rho_{\mathrm{eff}} a d r-V \frac{\dot{G}_{\mathrm{eff}}}{G_{\mathrm{eff}}} \rho_{\mathrm{eff}} d t .
\end{aligned}
$$

By the replacement $a d r=d \Upsilon-H \Upsilon d t$, Eqs.(83) and (84) can be recast into the $(t, \Upsilon)$ transverse coordinates as

$$
\begin{aligned}
d E_{\mathrm{eff}} & =-\frac{\dot{\Upsilon}_{\mathrm{A}}}{G_{\mathrm{eff}}} \frac{\Upsilon^{3}}{\Upsilon_{\mathrm{A}}^{3}} d t+\frac{3}{2 G_{\mathrm{eff}}} \frac{\Upsilon^{2}}{\Upsilon_{\mathrm{A}}^{2}} d \Upsilon-\frac{\dot{G}_{\mathrm{eff}}}{2 G_{\mathrm{eff}}^{2}} \frac{\Upsilon^{3}}{\Upsilon_{\mathrm{A}}^{2}} d t \\
& =-A\left(1+w_{\mathrm{eff}}\right) \rho_{\mathrm{eff}} H \Upsilon d t+A \rho_{\mathrm{eff}} d \Upsilon-V \frac{\dot{G}_{\mathrm{eff}}}{G_{\mathrm{eff}}} \rho_{\mathrm{eff}} d t
\end{aligned}
$$

Both Eqs.(84) and (86) can be compactified into the thermodynamic equation

$$
d E_{\mathrm{eff}}=A \Psi+\mathcal{W} d V+\mathcal{E},
$$

where $\mathcal{W}$ and $\Psi$ respectively refer to the effective work density and the effective energy supply covector,

$$
\begin{gathered}
W=\frac{1}{2}\left(1-w_{\mathrm{eff}}\right) \rho_{\mathrm{eff}}, \\
\Psi=-\frac{1}{2}\left(1+w_{\mathrm{eff}}\right) \rho_{\mathrm{eff}} H \Upsilon d t+\frac{1}{2}\left(1+w_{\mathrm{eff}}\right) \rho_{\mathrm{eff}} a d r \\
=-\left(1+w_{\mathrm{eff}}\right) \rho_{\mathrm{eff}} H \Upsilon d t+\frac{1}{2}\left(1+w_{\mathrm{eff}}\right) \rho_{\mathrm{eff}} d \Upsilon
\end{gathered}
$$

and similar to Sec. III.1, $\mathcal{W}$ and $\Psi$ can trace back to the Hayward-type invariants $\mathcal{W}:=-\frac{1}{2} T_{(\mathrm{eff})}^{\alpha \beta} h_{\alpha \beta}$ and $\Psi_{\alpha}:=$ $T_{\alpha(\mathrm{eff})}^{\beta} \partial_{\beta} \Upsilon+\mathcal{W} \partial_{\alpha} \Upsilon$ under spherical symmetry. The $\mathcal{E}$ in Eq.(87) is an extensive energy term

$$
\mathcal{E}:=-V \frac{\dot{G}_{\mathrm{eff}}}{G_{\mathrm{eff}}} \rho_{\mathrm{eff}} d t .
$$

As will be shown in the next subsection, $\mathcal{E}$ contributes to the irreversible extra entropy production, so we regard Eq.(87) as the unified first law of nonequilibrium thermodynamics [64], which is an extension of the equilibrium version Eq.(23) in GR. Moreover, it follows from the contracted Bianchi identities and Eq.(75) that $\nabla_{\mu} G^{\mu}{ }_{v}=0=8 \pi \nabla_{\mu}\left(G_{\text {eff }} T_{\nu}^{\mu(\text { eff })}\right)$, and for the FRW metric Eq.(1) it leads to

$$
\dot{\rho}_{\mathrm{eff}}+3 H\left(\rho_{\mathrm{eff}}+P_{\mathrm{eff}}\right)=\frac{\dot{\mathcal{E}}}{V}=-\frac{\dot{G}_{\mathrm{eff}}}{G_{\mathrm{eff}}} \rho_{\mathrm{eff}},
$$

so $\mathcal{E}$ also shows up in the generalized continuity equation as a density dissipation effect.

\section{VI.3. Nonequilibrium Clausius equation on the horizon}

The holographic-style dynamical equation (79) can be slightly rearranged into $\frac{\dot{\Upsilon}_{\mathrm{A}}}{G_{\text {eff }}} d t=A_{\mathrm{A}}\left(1+w_{\text {eff }}\right) \rho_{\mathrm{eff}} H \Upsilon_{\mathrm{A}} d t$, so we have

$$
\begin{aligned}
& \frac{1}{2 \pi \Upsilon_{\mathrm{A}}} \cdot 2 \pi \Upsilon_{\mathrm{A}}\left(\frac{\dot{\Upsilon}_{\mathrm{A}}}{G_{\mathrm{eff}}} d t-\frac{1}{2} \Upsilon_{\mathrm{A}} \frac{\dot{G}_{\mathrm{eff}}}{G_{\mathrm{eff}}^{2}} d t\right)+ \\
& \frac{1}{2 \pi \Upsilon_{\mathrm{A}}} \cdot 2 \pi \Upsilon_{\mathrm{A}}\left(\frac{1}{2} \Upsilon_{\mathrm{A}} \frac{\dot{G}_{\mathrm{eff}}}{G_{\mathrm{eff}}^{2}} d t+V_{\mathrm{A}} \frac{\dot{G}_{\mathrm{eff}}}{G_{\mathrm{eff}}} \rho_{\mathrm{eff}} d t\right) \\
& =A_{\mathrm{A}}\left(1+w_{\mathrm{eff}}\right) \rho_{\mathrm{eff}} H \Upsilon_{\mathrm{A}} d t+V_{\mathrm{A}} \frac{\dot{G}_{\mathrm{eff}}}{G_{\mathrm{eff}}} \rho_{\mathrm{eff}} d t
\end{aligned}
$$

It can be formally compactified into the thermodynamic relation

$$
T_{\mathrm{A}}\left(d S_{\mathrm{A}}+d_{p} S^{(\mathrm{A})}\right)=-\left(A_{\mathrm{A}} \Psi_{t}+\mathcal{E}_{\mathrm{A}}\right)=-\left.d E_{\mathrm{eff}}^{\mathrm{A}}\right|_{d \Upsilon=0}
$$

where $\Psi_{t}$ is just the $t$-component of the covector $\Psi$ in Eq.(89), $\mathcal{E}_{\mathrm{A}}$ is the energy dissipation term Eq.(90) evaluated at $\Upsilon_{\mathrm{A}}$, and $T_{\mathrm{A}}=\frac{1}{2 \pi \Upsilon_{\mathrm{A}}}$ denotes the Cai-Kim temperature on $\Upsilon_{\mathrm{A}}$. Here $S_{\mathrm{A}}$ refers to the geometrically defined Wald entropy [65] for the dynamical apparent horizon,

$$
S_{\mathrm{A}}=\frac{\pi \Upsilon_{\mathrm{A}}^{2}}{G_{\mathrm{eff}}}=\frac{A_{\mathrm{A}}}{4 G_{\mathrm{eff}}}=\int \frac{d A_{\mathrm{A}}}{4 G_{\mathrm{eff}}},
$$

where $S_{\mathrm{A}}$ takes such a compact form due to $\Upsilon_{\mathrm{A}}=\Upsilon_{\mathrm{A}}(t)$ and $G_{\text {eff }}=G_{\text {eff }}(t)$ under the maximal spatial symmetry of the Universe, while $d_{p} S^{(\mathrm{A})}$ represents the irreversible entropy production within $\Upsilon_{\mathrm{A}}$

$$
\begin{aligned}
d_{p} S^{(\mathrm{A})} & =2 \pi \Upsilon_{\mathrm{A}}\left(\frac{1}{2} \Upsilon_{\mathrm{A}} \frac{\dot{G}_{\mathrm{eff}}}{G_{\mathrm{eff}}^{2}} d t+V_{\mathrm{A}} \frac{\dot{G}_{\mathrm{eff}}}{G_{\mathrm{eff}}} \rho_{\mathrm{eff}} d t\right) \\
& =2 \pi \Upsilon_{\mathrm{A}}^{2} \frac{\dot{G}_{\mathrm{eff}}}{G_{\mathrm{eff}}^{2}} d t
\end{aligned}
$$

where we have applied the following replacement

$$
\frac{1}{2} \Upsilon_{\mathrm{A}} \frac{\dot{G}_{\mathrm{eff}}}{G_{\mathrm{eff}}^{2}}=V_{\mathrm{A}} \frac{\dot{G}_{\mathrm{eff}}}{G_{\mathrm{eff}}} \rho_{\mathrm{eff}},
$$

whose validity is guaranteed by Eq.(78). Due to the extra entropy production element $d_{p} S^{(\mathrm{A})}$, we regard Eq.(93) as the nonequilibrium Clausius equation, which depicts the heat transfer plus the extensive energy dissipation for the isochoric process of an arbitrary instantaneous $\Upsilon_{\mathrm{A}}$. With the nonequilibrium unified first law Eq.(87), Eq.(93) can be completed into the total energy differential

$$
\begin{aligned}
d E_{\mathrm{eff}}^{\mathrm{A}} & =A_{\mathrm{A}} \Psi_{t} d t+A_{\mathrm{A}}\left(\Psi_{\Upsilon}+\mathcal{W}\right) d \Upsilon_{\mathrm{A}}+\mathcal{E}_{\mathrm{A}} \\
& =-T_{\mathrm{A}}\left(d S_{\mathrm{A}}+d_{p} S^{(\mathrm{A})}\right)+\rho_{\mathrm{eff}} d V_{\mathrm{A}} .
\end{aligned}
$$




\section{VI.4. The second law for the interiors of the apparent and the event horizons}

For the cosmic entropy evolution, the second law of thermodynamics should still apply to the physical matter content $\left\{\rho_{m}, P_{m}\right\}$ rather than the mathematically effective $\left\{\rho_{\text {eff }}, P_{\text {eff }}\right\}$. Under minimal geometry-matter couplings, the Noether compatible definition of $T_{\mu \nu}^{(m)}$ automatically guarantees $\nabla^{\mu} T_{\mu \nu}^{(m)}=$ 0 , so the total continuity equation (91) can be decomposed into the ordinary one for the physical matter and the remaining part for the modified-gravity effect [64]:

$$
\begin{aligned}
\dot{\rho}_{m}+3 H\left(\rho_{m}+P_{m}\right) & =0 \\
\dot{\rho}_{(\mathrm{MG})}+3 H\left(\rho_{(\mathrm{MG})}+P_{(\mathrm{MG})}\right) & =-\frac{\dot{G}_{\mathrm{eff}}}{G_{\mathrm{eff}}}\left(\rho_{m}+\rho_{(\mathrm{MG})}\right) .
\end{aligned}
$$

For the physical energy $E_{m}=\rho_{m} V=E_{\text {eff }}-\rho_{(\mathrm{MG})} V$ within an arbitrary volume, the positive-heat-out Gibbs equation (54) still yields $T_{m} d S_{m}=-d\left(\rho_{m} V\right)-P_{m} d V=-V d \rho_{m}-\left(\rho_{m}+\right.$ $\left.P_{m}\right) d V$, which together with Eq.(98) leads to

$$
\begin{aligned}
T_{m} d S_{m} & =3 H\left(\rho_{m}+P_{m}\right) V d t-\left(\rho_{m}+P_{m}\right) A d \Upsilon \\
& =\rho_{m} A\left(1+w_{m}\right)(H \Upsilon d t-d \Upsilon) .
\end{aligned}
$$

Hence, for the physical entropy $S_{m}^{(\mathrm{A})}$ inside the apparent horizon $\Upsilon_{\mathrm{A}}(t)$, Eq.(99) and the holographic-style dynamical equation (80) yield

$$
\begin{aligned}
T_{m} d S_{m}^{(\mathrm{A})} & =\rho_{m} A_{\mathrm{A}}\left(1+w_{m}\right)\left(\Upsilon_{\mathrm{A}} H-\dot{\Upsilon}_{\mathrm{A}}\right) d t \\
& =-\frac{3}{2} \rho_{m} A_{\mathrm{A}}\left(1+w_{m}\right) H \Upsilon_{\mathrm{A}}\left(\frac{1}{3}+w_{\mathrm{eff}}\right) d t \\
& =-\frac{9}{2} \rho_{m} V_{\mathrm{A}} H\left(1+w_{m}\right)\left(\frac{1}{3}+w_{\mathrm{eff}}\right) d t .
\end{aligned}
$$

where $\rho_{m} A_{\mathrm{A}}$ cannot be simplified by Eq.(10) of GR. Recall that $-1 / 3 \leq w_{m} \leq 1$ in modified gravities, thus:

Theorem 4 The physical entropy $S_{m}^{(A)}$ inside the cosmological apparent horizon satisfies $\dot{S}_{m}^{(A)} \geq 0$ only when $w_{\text {eff }} \leq-1 / 3$.

Moreover, inside the event horizon $\Upsilon_{\mathrm{E}}(t)$, Eq.(99) along with $\dot{\Upsilon}_{\mathrm{E}}=H \Upsilon_{\mathrm{E}}-1$ give rise to

$$
\begin{aligned}
T_{m} d S_{m}^{(\mathrm{E})} & =\rho_{m} A_{\mathrm{E}}\left(1+w_{m}\right)\left(H \Upsilon_{\mathrm{E}}-\dot{\Upsilon}_{\mathrm{E}}\right) d t \\
& =\rho_{m} A_{\mathrm{E}}\left(1+w_{m}\right) d t .
\end{aligned}
$$

Hence, for the FRW Universe governed by modified gravities and filled with ordinary matter $-1 / 3 \leq w_{m} \leq 1$ :

Theorem 5 The physical entropy $S_{m}^{(E)}$ inside the cosmological event horizon always satisfies $\dot{S}_{m}^{(E)}>0$ regardless of the modified-gravity theories in use.

\section{VI.5. GSL for the apparent-horizon system}

Unlike the standard second law for the matter content $\left\{\rho_{m}, P_{m}\right\}$, GSLs further involve the modified-gravity effects $\left\{\rho_{(\mathrm{MG})}, P_{(\mathrm{MG})}\right\}$ which influence the horizon entropy. Compared with the $\Lambda \mathrm{CDM}$ situation in Sec. V.5, there are three types of entropy for the apparent-horizon system in modified gravities: the physical $S_{m}^{(\mathrm{A})}$ for the internal matter content, the Wald entropy $S_{\mathrm{A}}$ of the horizon $\Upsilon_{\mathrm{A}}$, and the nonequilibrium extensive entropy production. From Eqs.(93) and (100), we have

$$
\begin{aligned}
& \dot{S}_{m}^{(\mathrm{A})}+\dot{S}_{\mathrm{A}}+\dot{S}_{p}^{(\mathrm{A})} \\
= & -\frac{1}{T_{m}} \frac{3}{2} \rho_{m} A_{\mathrm{A}}\left(1+w_{m}\right) H \Upsilon_{\mathrm{A}}\left(\frac{1}{3}+w_{\mathrm{eff}}\right) \\
& +\frac{2 \pi \Upsilon_{\mathrm{A}} \dot{\Upsilon}_{\mathrm{A}}}{G_{\mathrm{eff}}}+\pi \Upsilon_{\mathrm{A}}^{2} \frac{\dot{G}_{\mathrm{eff}}}{G_{\mathrm{eff}}^{2}} \\
= & \frac{3}{2} \frac{\Upsilon_{\mathrm{A}}}{\Upsilon_{\mathrm{H}}}\left(-\frac{1}{T_{m}} \rho_{m} A_{\mathrm{A}}\left(1+w_{m}\right)\left(\frac{1}{3}+w_{\mathrm{eff}}\right)+\frac{1}{T_{\mathrm{A}}} \frac{1}{G_{\mathrm{eff}}}\left(1+w_{\mathrm{eff}}\right)\right. \\
& \left.+\frac{1}{T_{\mathrm{A}}} \frac{1}{3 H} \frac{\dot{G}_{\mathrm{eff}}}{G_{\mathrm{eff}}^{2}}\right),
\end{aligned}
$$

where $\dot{S}_{p}^{(\mathrm{A})}:=d_{p} S^{(\mathrm{A})} / d t, T_{\mathrm{A}}=1 /\left(2 \pi \Upsilon_{\mathrm{A}}\right)$, and $\dot{\Upsilon}_{\mathrm{A}}=$ $\frac{3}{2} H \Upsilon_{\mathrm{A}}\left(1+w_{\text {eff }}\right)$. Generally the GSL for the apparent-horizon system does not hold because the region $\Upsilon \leq \Upsilon_{\mathrm{A}}$ only comprises a finite portion of the Universe and is thermodynamically open with the absolute Hubble flow crossing $\Upsilon_{\mathrm{A}}$. However, Eq.(102) shows that $\dot{S}_{m}^{(\mathrm{A})}+\dot{S}_{\mathrm{A}}+\dot{S}_{p}^{(\mathrm{A})} \geq 0$ could validate when

$$
\frac{T_{m}}{T_{\mathrm{A}}}\left(\frac{1+w_{\mathrm{eff}}}{G_{\mathrm{eff}}}+\frac{1}{3 H} \frac{\dot{G}_{\mathrm{eff}}}{G_{\mathrm{eff}}^{2}}\right) \geq \rho_{m} A_{\mathrm{A}}\left(1+w_{m}\right)\left(\frac{1}{3}+w_{\mathrm{eff}}\right),
$$

where $A_{\mathrm{A}}$ cannot be further replaced by $1 /\left(\pi T_{\mathrm{A}}^{2}\right)$ to nonlinearize $T_{\mathrm{A}}$ since $T_{\mathrm{A}}$ is not an extensive quantity. Specifically for equilibrium theories with $G_{\text {eff }}=$ constant, like the dynamical Chern-Simons gravity [61, 64], Eq.(103) reduces to become

$$
\left(1+w_{\mathrm{eff}}\right) \frac{T_{m}}{T_{\mathrm{A}}} \geq \rho_{m} A_{\mathrm{A}} G\left(1+w_{m}\right)\left(\frac{1}{3}+w_{\mathrm{eff}}\right),
$$

which appears analogous to Eq.(63) of $\Lambda \mathrm{CDM}$.

For the apparent-horizon GSL, these results have matured the pioneering investigations in [18] for generic modified gravities and other earlier results in e.g. [22, 24] for specific gravity theories by the nonequilibrium revision of the unified first law, selection of the Cai-Kim temperature, dropping of the artificial assumption $T_{m}=\mathcal{T}_{\mathrm{A}}^{(+)}$, and discovery of the explicit expression for the entropy production $d_{p} S^{(\mathrm{A})}$.

\section{VI.6. GSL for the event-horizon system}

For the event-horizon system, $d S_{\mathrm{E}}+d_{p} S^{(\mathrm{E})}$ should be directly determined by the nonequilibrium unified first law 
Eq.(86),

$$
\begin{aligned}
& T_{\mathrm{E}}\left(d S_{\mathrm{E}}+d_{p} S^{(\mathrm{E})}\right) \\
= & \delta Q_{(\mathrm{E})}=-\left.d E_{\mathrm{eff}}^{(\mathrm{E})}\right|_{\Upsilon_{\mathrm{E} 0}}=-\left(A_{\mathrm{E}} \Psi_{t}+\mathcal{E}_{\mathrm{E}}\right) \\
= & A_{\mathrm{E}}\left(1+w_{\mathrm{eff}}\right) \rho_{\mathrm{eff}} H \Upsilon_{\mathrm{E}} d t+V_{\mathrm{E}} \frac{\dot{G}_{\mathrm{eff}}}{G_{\mathrm{eff}}} \rho_{\mathrm{eff}} d t .
\end{aligned}
$$

Then Eqs.(101) and (105) yield

$$
\begin{aligned}
& \dot{S}_{m}^{(\mathrm{E})}+\dot{S}_{\mathrm{E}}+\dot{S}_{p}^{(\mathrm{E})}=\frac{1}{T_{m}} \rho_{m} A_{\mathrm{E}}\left(1+w_{m}\right)+ \\
& \frac{1}{T_{\mathrm{E}}}\left(A_{\mathrm{E}}\left(1+w_{\mathrm{eff}}\right) \rho_{\mathrm{eff}} H \Upsilon_{\mathrm{E}}+V_{\mathrm{E}} \frac{\dot{G}_{\mathrm{eff}}}{G_{\mathrm{eff}}} \rho_{\mathrm{eff}}\right) .
\end{aligned}
$$

Inspired by the validity of the event-horizon GSL for Sec. V.6 and the standard second law Eq.(101), we a priori anticipate $\dot{S}_{m}^{(\mathrm{E})}+\dot{S}_{\mathrm{E}}+\dot{S}_{p}^{(\mathrm{E})} \geq 0$ to hold, which imposes the following viability constraint to modified gravities

$$
\frac{T_{m}}{T_{\mathrm{E}}}\left(\left(1+w_{\mathrm{eff}}\right) H+\frac{\dot{G}_{\text {eff }}}{G_{\text {eff }}}\right) \rho_{\text {eff }} \geq-\rho_{m}\left(1+w_{m}\right) \Upsilon_{\mathrm{E}}^{-1} .
$$

Considering that $-1 / 3 \leq w_{m} \leq 1$, its right hand side is negative definite, so a sufficient (yet not necessary) condition to validate the GSL is

$$
\left(\left(1+w_{\text {eff }}\right) H+\frac{\dot{G}_{\text {eff }}}{G_{\text {eff }}}\right) \rho_{\text {eff }} \geq 0 .
$$

These results improve the earlier investigations in e.g. [56] for the event-horizon GSL in modified gravities.

Note that the discussion in Sec. VI.3 is based on the holographic-style gravitational equations and only applies to the apparent-horizon system; if presuming a Wald entropy $A_{\mathrm{E}} / 4 G_{\text {eff }}$ and employing the entropy production to balance all differential terms involving the evolution effect $\dot{G}_{\text {eff }}$, one would obtain

$$
T_{\mathrm{E}}\left(d S_{\mathrm{E}}+d_{p} S^{(\mathrm{E})}\right)=\left(T_{\mathrm{E}} \frac{2 \pi \Upsilon_{\mathrm{E}} \dot{\Upsilon}_{\mathrm{E}}}{G_{\text {eff }}}+V_{\mathrm{E}} \frac{\dot{G}_{\text {eff }}}{G_{\text {eff }}} \rho_{\text {eff }}\right) d t,
$$

with $d_{p} S^{(\mathrm{E})}$ specified as

$$
d_{p} S^{(\mathrm{E})}=\left(T_{\mathrm{E}} A_{\mathrm{E}} \frac{\dot{G}_{\text {eff }}}{4 G_{\text {eff }}^{2}}+V_{\mathrm{E}} \frac{\dot{G}_{\text {eff }}}{G_{\text {eff }}} \rho_{\text {eff }}\right) d t .
$$

Comparison of Eqs.(105) and (109) yields the condition

$$
T_{\mathrm{E}} \frac{2 \pi \Upsilon_{\mathrm{E}}\left(H \Upsilon_{\mathrm{E}}-1\right)}{G_{\mathrm{eff}}}=A_{\mathrm{E}}\left(1+w_{\mathrm{eff}}\right) \rho_{\mathrm{eff}} H \Upsilon_{\mathrm{E}}
$$

and thus the whole discussion in Sec. V.7 for $\Lambda$ CDM can be parallelly applied to modified gravities with $G \mapsto G_{\text {eff }}$, $\rho_{m} \mapsto \rho_{\text {eff }}$ and $w_{m} \mapsto w_{\text {eff }}$, which again implies that the entropy $A / 4 G_{\text {eff }}$ and the Cai-Kim temperature $1 /(2 \pi \Upsilon)$ only unconditionally hold on the cosmological event horizon.

\section{VI.7. A note on existing methods of GSL}

Existent papers on GSL of modified gravities (in the traditional positive-heat-in Gibbs equation $T_{m} d \widehat{S}_{m}=d E+P_{m} d V$ ) usually replace $\rho_{m}+P_{m}$ by $\widetilde{\rho}_{(\mathrm{MG})}+\widetilde{P}_{(\mathrm{MG})}$ in Eq.(99), with $\left\{\widetilde{\rho}_{(\mathrm{MG})}, \widetilde{P}_{(\mathrm{MG})}\right\}$ set up in the field equation involving both Newton's constant $G$ and the dynamic $G_{\text {eff: }}$ :

$$
R_{\mu \nu}-\frac{1}{2} R g_{\mu \nu}=8 \pi G \widetilde{T}_{\mu \nu}^{(\mathrm{eff})}=8 \pi G\left(\widetilde{T}_{\mu \nu}^{(m)}+\widetilde{T}_{\mu \nu}^{(\mathrm{MG})}\right),
$$

where $\widetilde{T}^{\mu(\mathrm{eff})}=\operatorname{diag}\left[-\widetilde{\rho}_{\text {eff }}, \widetilde{P}_{\text {eff }}, \widetilde{P}_{\text {eff }}, \widetilde{P}_{\text {eff }}\right], \widetilde{\rho}_{\text {eff }}=\widetilde{\rho}_{m}+\widetilde{\rho}_{(\mathrm{MG})}$, $\widetilde{P}_{\text {eff }}=\widetilde{P}_{m}+\widetilde{P}_{(\mathrm{MG})}$, and the tilde $\sim$ means that the possibly dynamical aspect of $G_{\text {eff }}$ in Eq.(75) has been absorbed into $\widetilde{T}_{\mu \nu}^{\text {(eff) }}$ to formally maintain a constant coupling strength $G$; also note that for these tilded quantities the conservation equation becomes $\dot{\tilde{\rho}}_{\text {eff }}+3 H\left(\tilde{\rho}_{\text {eff }}+\tilde{P}_{\text {eff }}\right)=0$ and $\dot{\rho}_{m}+3 H\left(\rho_{m}+P_{m}\right)=0$ under minimal coupling (an energy exchange term between $\rho_{m}$ and $\widetilde{\rho}_{(\mathrm{MG})}$ was analyzed for minimal $f(R)$ gravity in [25], which however should be a feature of nonminimal coupling). This way, for the apparent-horizon system with $T_{m} \dot{\bar{S}}_{m}=$ $4 \pi \Upsilon_{\mathrm{A}}^{2}\left(\rho_{m}+P_{m}\right)\left(\Upsilon_{\mathrm{A}}-H \dot{\Upsilon}_{\mathrm{A}}\right) d t$, one would have the GSL (e.g. $[22,24,25]$ for the $F(R, \mathcal{G})$, scalar-tensor-chameleon and interacting $f(R)$ gravities)

$$
\dot{S}_{m}^{(\mathrm{A})}+\dot{S}_{\mathrm{A}}=\frac{1}{T_{m}} \frac{G}{G_{\mathrm{eff}}}\left(\frac{\dot{\Upsilon}_{\mathrm{A}}}{G H \Upsilon_{\mathrm{A}}}-4 \pi \Upsilon_{\mathrm{A}}^{2}\left(\widetilde{\rho}_{(\mathrm{MG})}+\widetilde{P}_{(\mathrm{MG})}\right)\right)\left(\dot{\Upsilon}_{\mathrm{A}}-\Upsilon_{\mathrm{A}} H\right)+\frac{2 \pi \Upsilon_{\mathrm{A}} \dot{\Upsilon}_{\mathrm{A}}}{G_{\mathrm{eff}}}
$$

where $G_{\text {eff }}$ is recognized from the coefficient of $G \widetilde{\rho}_{m}=G_{\text {effi }} \rho_{m}$ to utilize the Wald entropy $S_{\mathrm{A}}=A_{\mathrm{A}} / 4 G_{\text {eff. }}$. In Eq.(113) we have incorporated the holographic-style gravitational equations [simply Eqs.(78)-(81) with $G_{\text {eff }} \mapsto G$ and $\rho_{\text {eff }} \mapsto \widetilde{\rho}_{\text {eff }}, P_{\text {eff }} \mapsto \widetilde{P}_{\text {eff] }}$ for compactness, as well as the relation

$$
\rho_{m}+P_{m}=\frac{G}{G_{\mathrm{eff}}}\left(\widetilde{\rho}_{m}+\widetilde{P}_{m}\right) .
$$

However, Eq.(113) is not self-consistent, not just for the conflicting sign conventions encoded in $\widehat{S}_{m}^{(\mathrm{A})}$ and $S_{\mathrm{A}}$, but also because it uses two different coupling strength for $\left\{\widehat{S}_{m}^{(\mathrm{A})}, S_{\mathrm{A}}\right\}$, and fails to capture the extra entropy production $d_{p} S^{(\mathrm{A})}$ which arises in all modified gravities with nontrivial $G_{\text {eff }}[64,67]$. To overcome these flaws in this popular method, the adjusted Gibbs equation 
(54) along with the setups in Eqs. $(75,76)$ and the holographic-style Eqs.(78)-(81) lead to

$$
\begin{aligned}
T_{m} \dot{S}_{m} & =-\frac{1}{G_{\mathrm{eff}}}\left(\frac{\dot{\Upsilon}_{\mathrm{A}}}{H \Upsilon_{\mathrm{A}}}-4 \pi \Upsilon_{\mathrm{A}}^{2} G_{\mathrm{eff}}\left(\rho_{(\mathrm{MG})}+P_{(\mathrm{MG})}\right)\right)\left(\dot{\Upsilon}_{\mathrm{A}}-\Upsilon_{\mathrm{A}} H\right) \\
& =-\frac{H \Upsilon_{\mathrm{A}}^{5}}{G_{\mathrm{eff}}}\left(\dot{H}-\frac{k}{a^{2}}+4 \pi G_{\mathrm{eff}}\left(\rho_{(\mathrm{MG})}+P_{(\mathrm{MG})}\right)\right)\left(\dot{H}+H^{2}\right),
\end{aligned}
$$

which together with Eq.(93) yields

$$
\dot{S}_{m}^{(\mathrm{A})}+\dot{S}_{\mathrm{A}}+\dot{S}_{p}^{(\mathrm{A})}=-\frac{H \Upsilon_{\mathrm{A}}^{5}}{G_{\mathrm{eff}}}\left(\dot{H}-\frac{k}{a^{2}}+4 \pi G_{\mathrm{eff}}\left(\rho_{(\mathrm{MG})}+P_{(\mathrm{MG})}\right)\right)\left(\dot{H}+H^{2}\right)+\frac{2 \pi \Upsilon_{\mathrm{A}} \dot{\Upsilon}_{\mathrm{A}}}{G_{\mathrm{eff}}}+\pi \Upsilon_{\mathrm{A}}^{2} \frac{\dot{G}_{\mathrm{eff}}}{G_{\mathrm{eff}}^{2}} .
$$

Eq.(116) improves Eq.(113) into a totally self-consistent and more natural method that employs a single gravitational coupling strength $G_{\text {eff }}$ in accordance with the standard entropy $A_{\mathrm{A}} / 4 G_{\text {eff. }}$. The approach by Eq.(116) looks more concentrative on $\left\{\rho_{(\mathrm{MG})}, P_{(\mathrm{MG})}\right\}$ of the modified-gravity effects; however, it has implicitly ignored the nonexotic character of the cosmic fluid $\rho_{m}+3 P_{m} \geq 0$, and complicated the mathematical calculations. Hence, in this paper we have chosen to work with Eqs. $(100,102)$ rather than Eqs. $(115,116)$ for the apparenthorizon system, and similarly Eq. $(101,106)$ for the eventhorizon system.

\section{VI.8. Applications to concrete modified gravities}

The formulation of gravitational thermodynamics in this section applies to all ordinary modified gravities of the form Eq.(75). One can just reverse the process and logic in [64] to see the detailed applications of the first laws for different gravity theories, and in this paper we will focus on the concretization of the second laws, for which we have drawn the following generic conclusions:

(1) $\dot{S}_{m}^{(\text {E) }}>0$ always holds, while $\dot{S}_{m}^{(\mathrm{A})} \geq 0$ when $w_{\text {eff }} \leq$ $-1 / 3$

(2) $\dot{S}_{m}^{(\mathrm{E})}+\dot{S}_{\mathrm{E}}+\dot{S}_{p}^{(\mathrm{E})} \geq 0$ should hold with Eq.(107) as a validity constraint for modified gravities, while $\dot{S}_{m}^{(\text {A) }}+\dot{S}_{\mathrm{A}}+$ $\dot{S}_{p}^{(\mathrm{A})} \geq 0$ could conditionally hold only when Eq.(103) is satisfied.

To concretize these conditions, one just needs to find out the effective gravitational coupling strength $G_{\text {eff }}$, the effective EoS parameter

$$
\begin{aligned}
w_{\mathrm{eff}} & =\frac{\left(P_{\mathrm{eff}}+\rho_{\mathrm{eff}}\right)-\rho_{\mathrm{eff}}}{\rho_{\mathrm{eff}}} \\
& =-1+\frac{\left(1+w_{m}\right) \rho_{m}+\left(\rho_{(\mathrm{MG})}+P_{(\mathrm{MG})}\right)}{\rho_{m}+\rho_{(\mathrm{MG})}},
\end{aligned}
$$

the "modified-gravity energy density" $\rho_{(\mathrm{MG})}$, and $\rho_{(\mathrm{MG})}+$ $P_{(\mathrm{MG})}$.

\section{VI.8.1. $\quad f(R)$ gravity}

For the FRW Universe governed by the $\mathscr{L}=f(R)+$ $16 \pi G \mathscr{L}_{m}$ gravity [59], we have $G_{\text {eff }}=G / f_{R}$ and [64]

$$
\begin{gathered}
\rho_{(\mathrm{MG})}=\frac{1}{8 \pi G}\left(\frac{1}{2} f_{R} R-\frac{1}{2} f-3 H \dot{f}_{R}\right) \\
\rho_{(\mathrm{MG})}+P_{(\mathrm{MG})}=\frac{1}{8 \pi G}\left(\ddot{f}_{R}-H \dot{f}_{R}\right) \\
w_{\mathrm{eff}}=-1+\frac{8 \pi G\left(1+w_{m}\right) \rho_{m}+\ddot{f}_{R}-H \dot{f}_{R}}{8 \pi G \rho_{m}+\frac{1}{2} f_{R} R-\frac{1}{2} f-3 H \dot{f}_{R}} .
\end{gathered}
$$

The GSL for the event-horizon system requires $f(R)$ gravity to respect the following viability condition

$$
\begin{aligned}
& \frac{T_{m}}{T_{\mathrm{E}}}\left(\frac{8 \pi G\left(1+w_{m}\right) \rho_{m}+\ddot{f}_{R}-H \dot{f}_{R}}{8 \pi G \rho_{m}+\frac{1}{2} f_{R} R-\frac{1}{2} f-3 H \dot{f}_{R}} H-\frac{\dot{f}_{R}}{f_{R}}\right) \times \\
& \left(8 \pi G \rho_{m}+\frac{1}{2} f_{R} R-\frac{1}{2} f-3 H \dot{f}_{R}\right) \geq-8 \pi G \rho_{m}\left(1+w_{m}\right) \Upsilon_{\mathrm{E}}^{-1},
\end{aligned}
$$

while for the apparent-horizon open system, the second law and the GSL respectively hold in the situations

$$
\begin{gathered}
\frac{8 \pi G\left(1+w_{m}\right) \rho_{m}+\ddot{f}_{R}-H \dot{f}_{R}}{8 \pi G \rho_{m}+\frac{1}{2} f_{R} R-\frac{1}{2} f-3 H \dot{f}_{R}} \leq-\frac{2}{3} \\
\frac{T_{m}}{T_{\mathrm{A}}}\left(f_{R} \frac{8 \pi G\left(1+w_{m}\right) \rho_{m}+\ddot{f}_{R}-H \dot{f}_{R}}{8 \pi G \rho_{m}+\frac{1}{2} f_{R} R-\frac{1}{2} f-3 H \dot{f}_{R}}-\frac{\dot{f_{R}}}{3 H}\right) \geq \\
G \rho_{m} A_{\mathrm{A}}\left(1+w_{m}\right)\left(\frac{8 \pi G\left(1+w_{m}\right) \rho_{m}+\ddot{f}_{R}-H \dot{f}_{R}}{8 \pi G \rho_{m}+\frac{1}{2} f_{R} R-\frac{1}{2} f-3 H \dot{f}_{R}}-\frac{2}{3}\right) .
\end{gathered}
$$

VI.8.2. Scalar-tensor-chameleon gravity

For the scalar-tensor-chameleon gravity [24] with the Lagrangian density $\mathscr{L}_{\text {STC }}=F(\phi) R-Z(\phi) \nabla_{\alpha} \phi \nabla^{\alpha} \phi-2 U(\phi)+$ $16 \pi G E(\phi) \mathscr{L}_{m}$, we have $G_{\text {eff }}=\frac{E(\phi)}{F(\phi)} G$ and [64]

$$
\rho_{(\mathrm{MG})}=\frac{1}{8 \pi G E}\left(-3 H \dot{F}+\frac{1}{2} Z \dot{\phi}^{2}+U\right)
$$




$$
w_{\mathrm{eff}}=-1+\frac{8 \pi G E\left(1+w_{m}\right) \rho_{m}+\ddot{F}-H \dot{F}+Z \dot{\phi}^{2}}{8 \pi G E \rho_{m}-3 H \dot{F}+\frac{1}{2} Z \dot{\phi}^{2}+U},
$$

where in this subsection we temporarily adopt the abbreviations $E \equiv E(\phi), F \equiv F(\phi), U \equiv U(\phi)$ and $Z \equiv Z(\phi)$. Eq. (107) for the GSL of the event-horizon system imposes the constraint

$$
\begin{aligned}
& \frac{T_{m}}{T_{\mathrm{E}}}\left(\frac{8 \pi G E\left(1+w_{m}\right) \rho_{m}+\ddot{F}-H \dot{F}+Z \dot{\phi}^{2}}{8 \pi G E \rho_{m}-3 H \dot{F}+\frac{1}{2} Z \dot{\phi}^{2}+U} H+\frac{F E_{\phi}-E F_{\phi}}{E F} \dot{\phi}\right) \\
& \times\left(8 \pi G \rho_{m}-3 H \frac{\dot{F}}{E}+\frac{Z}{E} \dot{\phi}^{2}+\frac{U}{E}\right) \geq-8 \pi G \rho_{m}\left(1+w_{m}\right) \Upsilon_{\mathrm{E}}^{-1},
\end{aligned}
$$

while $w_{\text {eff }} \leq-\frac{1}{3}$ and the apparent-horizon GSL Eq.(107) can be directly realized with Eq.(129) and $\rho_{(\mathrm{MG})}+P_{(\mathrm{MG})}=$ $\frac{1}{8 \pi G E}\left(\ddot{F}-H \dot{F}+Z \dot{\phi}^{2}\right)$. Moreover, in the specifications $E \mapsto 1$, $F \mapsto \phi, Z \mapsto \omega / \phi, U \mapsto \frac{1}{2} V$, we recover the generalized Brans-Dicke gravity [62] with a self-interacting potential, $\mathscr{L}_{\mathrm{GBD}}=\phi R-\frac{\omega}{\phi} \nabla_{\alpha} \phi \nabla^{\alpha} \phi-V(\phi)+16 \pi G \mathscr{L}_{m}$, and Eq.(130) reduces to become

$$
\begin{aligned}
& \frac{T_{m}}{T_{\mathrm{E}}}\left(\frac{8 \pi G\left(1+w_{m}\right) \rho_{m}+\ddot{\phi}-H \dot{\phi}+\frac{\omega}{\phi} \dot{\phi}^{2}}{8 \pi G \rho_{m}-3 H \dot{\phi}+\frac{\omega}{2 \phi} \dot{\phi}^{2}+\frac{1}{2} V} H-\frac{\dot{\phi}}{\phi}\right) \times \\
& \left(8 \pi G \rho_{m}-3 H \dot{\phi}+\frac{\omega}{2 \phi} \dot{\phi}^{2}+\frac{V}{2}\right) \geq-8 \pi G \rho_{m}\left(1+w_{m}\right) \Upsilon_{\mathrm{E}}^{-1} .
\end{aligned}
$$

where in this subsection we temporarily adopt the abbreviations $E \equiv E(\phi), F \equiv F(\phi), U \equiv U(\phi)$ and $Z \equiv Z(\phi)$, while $H$ is still the Hubble parameter. Eq.(107) for the GSL of the event-horizon system imposes the constraint

$$
\frac{T_{m}}{T_{\mathrm{E}}}\left(\frac{8 \pi G E\left(1+w_{m}\right) \rho_{m}+\ddot{F}-H \dot{F}+Z \dot{\phi}^{2}}{8 \pi G E \rho_{m}-3 H \dot{F}+\frac{1}{2} Z \dot{\phi}^{2}+U} H+\frac{F E_{\phi}-E F_{\phi}}{E F} \dot{\phi}\right) \times\left(8 \pi G \rho_{m}-3 H \frac{\dot{F}}{E}+\frac{Z}{2 E} \dot{\phi}^{2}+\frac{U}{E}\right) \geq-8 \pi G \rho_{m}\left(1+w_{m}\right) \mathrm{\Upsilon}_{\mathrm{E}}^{-1},
$$

while $w_{\text {eff }} \leq-\frac{1}{3}$ and the apparent-horizon GSL Eq.(107) can be directly realized with Eq.(129) and $\rho_{(\mathrm{MG})}+P_{(\mathrm{MG})}=$ $\frac{1}{8 \pi G E}\left(\ddot{F}-H \dot{F}+Z \dot{\phi}^{2}\right)$. Moreover, in the specifications $E \mapsto 1, F \mapsto \phi, Z \mapsto \omega / \phi, U \mapsto \frac{1}{2} V$, we recover the generalized Brans-Dicke gravity [62] with a self-interacting potential, $\mathscr{L}_{\mathrm{GBD}}=\phi R-\frac{\omega}{\phi} \nabla_{\alpha} \phi \nabla^{\alpha} \phi-V(\phi)+16 \pi G \mathscr{L}_{m}$, and Eq.(130) reduces to become

$$
\frac{T_{m}}{T_{\mathrm{E}}}\left(\frac{8 \pi G\left(1+w_{m}\right) \rho_{m}+\ddot{\phi}-H \dot{\phi}+\frac{\omega}{\phi} \dot{\phi}^{2}}{8 \pi G \rho_{m}-3 H \dot{\phi}+\frac{\omega}{2 \phi} \dot{\phi}^{2}+\frac{1}{2} V} H-\frac{\dot{\phi}}{\phi}\right) \times\left(8 \pi G \rho_{m}-3 H \dot{\phi}+\frac{\omega}{2 \phi} \dot{\phi}^{2}+\frac{1}{2} V\right) \geq-8 \pi G \rho_{m}\left(1+w_{m}\right) \Upsilon_{\mathrm{E}}^{-1}
$$

\section{VI.8.4. Quadratic gravity}

For the quadratic gravity $\mathscr{L}_{\mathrm{QG}}=R+a R^{2}+b R_{\mu \nu} R^{\mu \nu}+16 \pi G \mathscr{L}_{m}$ whose Lagrangian density is an effective linear superposition of the quadratic independent Riemannian invariants [58,60], with $\{a, b\}$ being constants, we have $G_{\text {eff }}=\frac{G}{1+2 a R}$ and [64]

$$
\begin{gathered}
\rho_{(\mathrm{MG})}=\frac{1}{8 \pi G}\left(\frac{a}{2} R^{2}-\frac{b}{2} R_{c}^{2}+\frac{b}{2} \ddot{R}-(4 a+b) H \dot{R}+4 b R_{\alpha t \beta}^{t}+2 b \square R_{t}{ }^{t}\right), \\
w_{\mathrm{eff}}=-1+\frac{8 \pi G\left(1+w_{m}\right) \rho_{m}+(2 a+b) \ddot{R}-\frac{b}{2} H \dot{R}+4 b\left(R_{\alpha t \beta}{ }_{\alpha \dot{ }}-R_{\alpha r \beta}^{r}\right) R^{\alpha \beta}+2 b \square\left(R_{t}{ }^{t}-R_{r}{ }^{r}\right)}{8 \pi G \rho_{m}+\frac{a}{2} R^{2}-\frac{b}{2} R_{c}^{2}+\frac{b}{2} \ddot{R}-(4 a+b) H \dot{R}+4 b R^{t}{ }_{\alpha t \beta}+2 b \square R_{t}{ }^{t}},
\end{gathered}
$$


where $R_{c}^{2}:=R_{\mu \nu} R^{\mu \nu}, \square=g^{\mu \nu} \nabla_{\mu} \nabla_{v}$, and we have used the compact geometric notations [64]. Hence, GSL of the event-horizon system requires

$$
\begin{aligned}
& \frac{T_{m}}{T_{\mathrm{E}}}\left(\frac{8 \pi G\left(1+w_{m}\right) \rho_{m}+(2 a+b) \ddot{R}-\frac{b}{2} H \dot{R}+4 b\left(R_{\alpha t \beta}^{t}-R_{\alpha r \beta}^{r}\right) R^{\alpha \beta}+2 b \square\left(R_{t}{ }^{t}-R_{r}{ }^{r}\right)}{8 \pi G \rho_{m}+\frac{a}{2} R^{2}-\frac{b}{2} R_{c}^{2}+\frac{b}{2} \ddot{R}-(4 a+b) H \dot{R}+4 b R_{\alpha t \beta}{ }^{t}+2 b \square R_{t}{ }^{t}} H-\frac{2 a \dot{R}}{1+2 a R}\right) \\
& \times\left(8 \pi G \rho_{m}+\frac{a}{2} R^{2}-\frac{b}{2} R_{c}^{2}+\frac{b}{2} \ddot{R}-(4 a+b) H \dot{R}+4 b R_{\alpha t \beta}^{t}+2 b \square R_{t}{ }^{t}\right) \geq-8 \pi G \rho_{m}\left(1+w_{m}\right) \Upsilon_{\mathrm{E}}^{-1},
\end{aligned}
$$

while $w_{\text {eff }} \leq-\frac{1}{3}$ and Eq.(107) can be directly concretized with Eq.(133) and

$$
\rho_{(\mathrm{MG})}+P_{(\mathrm{MG})}=\frac{1}{8 \pi G}\left((2 a+b) \ddot{R}-\frac{b}{2} H \dot{R}+4 b\left(R_{\alpha t \beta}^{t}-R_{\alpha r \beta}^{r}\right) R^{\alpha \beta}+2 b \square\left(R_{t}{ }^{t}-R_{r}{ }^{r}\right)\right) .
$$

\section{VI.8.5. Chern-Simons gravity}

Finally let's analyze the dynamical Chern-Simons gravity $\mathscr{L}_{\mathrm{CS}}=R+\frac{a \vartheta}{\sqrt{-g}} * \widehat{R R}-b \nabla_{\mu} \vartheta \nabla^{\mu} \vartheta-V(\vartheta)+16 \pi G \mathscr{L}_{m}$ [61] which has a constant gravitational coupling strength $G_{\text {eff }}=G$, where ${ }^{*} \widehat{R R}={ }^{*} R_{\alpha \beta \gamma \delta} R^{\alpha \beta \gamma \delta}$ denotes the Chern-Pontryagin invariant and $\{a, b\}$ are constants. We have [64]

$$
\begin{gathered}
\rho_{(\mathrm{MG})}=\frac{1}{16 \pi G}\left(b \dot{\vartheta}^{2}+V(\vartheta)\right) \\
w_{\mathrm{eff}}=-1+\frac{8 \pi G \rho_{m}\left(1+w_{m}\right)+b \dot{\vartheta}^{2}}{8 \pi G \rho_{m}+\frac{1}{2} b \dot{\vartheta}^{2}+\frac{1}{2} V(\vartheta)},
\end{gathered}
$$

and thus Eq.(107) leads to the viability condition

$$
\frac{T_{m}}{T_{\mathrm{E}}}\left(8 \pi G \rho_{m}\left(1+w_{m}\right)+b \dot{\vartheta}^{2}\right) \geq-8 \pi G \rho_{m}\left(1+w_{m}\right) \frac{\Upsilon_{\mathrm{H}}}{\Upsilon_{\mathrm{E}}},
$$

which, for $\dot{\vartheta} \neq 0$, yields a constraint for $b$,

$$
b \geq-8 \pi G \rho_{m}\left(1+w_{m}\right)\left(\frac{\Upsilon_{\mathrm{H}}}{\Upsilon_{\mathrm{E}}} \frac{T_{\mathrm{E}}}{T_{m}}+1\right) \dot{\vartheta}^{-2}
$$

For the FRW cosmology, ${ }^{*} \widehat{R R}$ makes no contribution to the gravitational equations, so $\mathscr{L}_{\mathrm{CS}}$ effectively acts as $\mathscr{L}=$ $R-b \nabla_{\mu} \vartheta \nabla^{\mu} \vartheta-V(\vartheta)+16 \pi G \mathscr{L}_{m}$, which formally resembles the scalarial dark energy [37, 39]. On the other hand, note that although Eqs.(138) and (139) are always satisfied for $b>0$, which corresponds to a canonical kinetic $\vartheta$-field that is quintessence-like $\left(\mathscr{L}=-\frac{1}{2} \nabla_{\mu} \phi \nabla^{\mu} \phi-V(\phi)\right), \vartheta$ is allowed to be slightly phantom-like $\left(\mathscr{L}=\frac{1}{2} \nabla_{\mu} \phi \nabla^{\mu} \phi-V(\phi)\right)$ for some $b<0$ by Eq.(139). Hence, Eq.(139) does not coincide with the situation of $\Lambda$ CDM in Sec. V.6, where $\dot{S}_{m}^{(\mathrm{E})}+\dot{S}_{\mathrm{E}} \geq 0$ holds if and only if $w_{m} \geq-1$.

\section{CONCLUSIONS AND DISCUSSION}

In this paper the thermodynamic implications of the holographic-style dynamical equations for the FRW Universe have been studied. We started from the $\Lambda$ CDM model of GR to clearly build the whole framework of gravitational thermo- dynamics, and eventually extended it to modified gravities. A great advantage of our formulation is all constraints are expressed by the EoS parameters.

The holographic-style gravitational equations govern both the apparent-horizon dynamics and the cosmic spatial expansion. We have shown how they imply Hayward's unified first law of equilibrium thermodynamics $d E=A \psi+W d V$ [46] and the isochoric-process Cai-Kim Clausius equation $T_{\mathrm{A}} d S_{\mathrm{A}}=$ $\delta Q_{\mathrm{A}}=-A_{\mathrm{A}} \psi_{t}[2,8,47]$. The derivations of the Clausius equation in Sec. III.2 actually involves a long standing confusion regarding the setup of the apparent-horizon temperature, and extensive comparisons in Sec. IV have led to the argument that the Cai-Kim $T_{\mathrm{A}}=1 /\left(2 \pi \Upsilon_{\mathrm{A}}\right)$ is more appropriate than the Hayward $\mathcal{T}_{\mathrm{A}}=\kappa / 2 \pi$ and its partial absolute value $\mathcal{T}_{\mathrm{A}}^{(+)}$. Meanwhile, we have also introduced the "zero temperature divide" $w_{m}=1 / 3$ for $\mathcal{T}_{\mathrm{A}}=\kappa / 2 \pi$, and proved the signs of both temperatures are independent of the inner or outer trappedness of the apparent horizon.

The "positive heat out" sign convention for the heat transfer and the horizon entropy has been decoded from $T_{\mathrm{A}} d S_{\mathrm{A}}=$ $-d E$, provided that the third law of thermodynamics holds with a positive $T_{\mathrm{A}}$. With the horizon temperature and entropy clarified, the cosmic entropy evolution has been investigated. We have adjusted the traditional matter entropy and Gibbs equation into $d E_{m}=-T_{m} d S_{m}-P_{m} d V_{\mathrm{A}}$ in accordance with the positive heat out convention of the horizon entropy. It turns out that the cosmic entropy is well behaved, specially for the event-horizon system, where both the second law and the GSL hold for nonexotic matter $\left(-1 \leq w_{m} \leq 1\right)$. Also, we have clarified that the regions $\left\{\Upsilon \leq \Upsilon_{A}, \Upsilon \leq \Upsilon_{E}\right\}$ enveloped by the apparent and even horizons are simple open thermodynamic systems $^{2}$ so that one should not a priori expect the validity of nondecreasing entropy, and abandoned the local equilibrium assumptions restricting the interior and the boundary temperatures.

Finally we have generalized the whole formulations from

\footnotetext{
${ }^{2}$ Even the philosophical "whole Universe" would be an open system if there were matter creations which would cause irreversible extra entropy production, and one typical mechanism triggering this effect is nonminimal curvature-matter coupling [66].
} 
the $\Lambda$ CDM model to ordinary modified gravities whose field equations have been intrinsically compactified into the GR form $R_{\mu \nu}-R g_{\mu \nu} / 2=8 \pi G_{\text {eff }} T_{\mu \nu}^{(\mathrm{eff})}$. To our particular interest, we found that inside the apparent horizon the second law $\dot{S}_{m} \geq 0$ nontrivially holds if $w_{\text {eff }} \leq-1 / 3$, while inside the event horizon $\dot{S}_{m} \geq 0$ always validates regardless of the gravity theories in use. These generic conclusions have been concretized in $f(R)$, scalar-tensor-chameleon, quadratic and dynamical Chern-Simons gravities.

Note that the volume $V$ and surface area $A$ used throughout this paper are interpreted as flat-space quantities in [26]. However, $\Upsilon$ and $A$ are the proper radius and area for the standard sphere $\mathbb{S}^{2}$ in the $2+2$ (rather than $3+1$ ) decomposition $d s^{2}=h_{\alpha \beta} d x^{\alpha} d x^{\beta}+\mathbb{S}^{2}$ of Eq.(1), while the role of $V$ as a proper quantity is still not clear.

There are still some interesting problems arising in this paper and yet unsolved. For example, the discussion in Sec. V.7 further raises the question that, what is the temperature $T_{\mathrm{E}}$ for the event horizon? Note that if $T_{\mathrm{E}} \neq T_{\mathrm{A}}$, there would be a spontaneous heat flow between $\Upsilon_{\mathrm{A}}$ and $\Upsilon_{\mathrm{E}}-$ would it affect the cosmic expansion? On the other hand, it is not clear whether or not the apparent and the event horizons could be heated by the absolute Hubble energy flow and consequently $T_{\mathrm{E}}=T_{m}$ and $T_{\mathrm{A}}=T_{m}$ : this would avoid the temperature gradient between $\Upsilon_{\mathrm{A}}$ and $\Upsilon_{\mathrm{E}}$, but throughout this paper we have not yet seen any evidence for $T_{\mathrm{A}}$ to be heated into $T_{m}$.

Moreover, besides the traditional GSLs, the "cosmic holographic principle" in [26] which argues that the physical entropy $\widehat{S}_{m}^{(\mathrm{A})}$ inside the apparent horizon $\Upsilon_{\mathrm{A}}$ could never exceed the apparent-horizon entropy $S_{\mathrm{A}}$, is also problematic in comparing $\widehat{S}_{m}^{(\mathrm{A})}$ with $S_{\mathrm{A}}$ - this principle should be restudied in the unified positive-heat-out sign convention. Moreover, is $\Upsilon_{\mathrm{A}}$ the only hologram membrane for the FRW Universe? Can the relative evolution equation (17) be used in astrophysical and cosmological simulations? Also, how would the cosmic entropy evolve in a contracting Universe? We hope to find out the answers in prospective studies.

\section{ACKNOWLEDGEMENT}

This work was financially supported by the Natural Sciences and Engineering Research Council of Canada.

\section{Appendix A: The minimum set of state functions and reversibility}

Eqs.(29) and (30) clearly indicate that just like ordinary thermodynamics, the geometrically defined horizon temperature $T_{\mathrm{A}}$ and horizon entropy $S_{\mathrm{A}}$ remain as state functions, which are independent of thermodynamic processes that indeed correspond to the details of cosmic expansion $\dot{a}(t)$ and the apparent-horizon evolution $\dot{\Upsilon}_{\mathrm{A}}$. Just like the regular tem- peratures of thermodynamic systems, the Cai-Kim $T_{\mathrm{A}}$ remains as an intensive property with $T_{\mathrm{A}}=T_{\mathrm{A}}(t)=1 / 2 \pi \Upsilon_{\mathrm{A}}(t)$; one should not treat it as an extensive property by $T_{\mathrm{A}}=$ $T_{\mathrm{A}}\left(V_{\mathrm{A}}\right)=1 /\left(2 \pi \sqrt[3]{\frac{3}{4 \pi} V_{\mathrm{A}}}\right)$. Some other state functions involved here include the apparent-horizon radius $\Upsilon_{\mathrm{A}}$, the energy density $\rho_{m}(t)$, the pressure $P_{m}(t)$ and thus the EoS parameter $w_{m}=\rho_{m} / P_{m}$. These state quantities are not totally independent as they are related with one another by the Friedmann equations (7), the holographic-style dynamical equation (8), and the thermodynamic relations in Secs. III.1 and III.2. Here we select the following quantities to comprise a minimum set of independent state functions for Secs. II and III:

$$
\text { Minimum set }=\left\{\rho_{m}, w_{m}, T_{\mathrm{A}}\right\} .
$$

Based on this set, the product of $\rho_{m}$ and $w_{m}$ yields the pressure $P_{m}$. Through Eq.(10) $\rho_{m}$ recovers the horizon area $A_{\mathrm{A}}$ and thus determines the entropy $S_{\mathrm{A}}$. Treating $T_{\mathrm{A}}$ as an intensive property, we do not take the approach from Eq.(8) or Eq.(10) for the recovery $\rho_{m} \rightarrow A_{\mathrm{A}} \rightarrow \Upsilon_{\mathrm{A}} \rightarrow T_{\mathrm{A}}$, and instead let $T_{\mathrm{A}}$ enter the minimum set directly as the Cai-Kim temperature ansatz. Similarly, for modified gravities with the dynamical equations (78)-(81), we choose the minimum set to be $\left\{\rho_{\text {eff }}, w_{\text {eff }}, G_{\text {eff }}, T_{\mathrm{A}}\right\}$.

The fact that Eq.(31) is the Clausius equation for (quasi)equilibrium or reversible thermodynamic processes without extra entropy production raises the question that, what does reversibility mean from the perspective of cosmic and apparent-horizon dynamics? From the explicit expression of the heat transfer $\delta Q_{\mathrm{A}}=T_{\mathrm{A}} d S_{\mathrm{A}}=A_{\mathrm{A}}\left(1+w_{m}\right) \rho_{m} H \Upsilon_{\mathrm{A}} d t$ where the state quantity $T_{\mathrm{A}} d S_{\mathrm{A}}$ is balanced by the process quantity $\delta Q_{\mathrm{A}}$, we naturally identify $H$ as a process quantity; moreover, if one reverses the initial and final states of $T_{\mathrm{A}} d S_{\mathrm{A}}$, the state quantities $\left\{\rho_{m}(t), w_{m}, \Upsilon_{\mathrm{A}}, A_{\mathrm{A}}\right\}$ can be automatically reversed. Hence, by reversibility we mean an imaginary negation $-H$ of the Hubble parameter that results in a spatial contraction process which directly evolves the Universe from a later state back to the earlier state of $T_{\mathrm{A}} d S_{\mathrm{A}}$ without reversing the time arrow and causing energy dissipation.

[68] suggests that since the energy-matter crossing the apparent horizon for the (accelerated) expanding Universe will not come back in the future, it should cause extra entropy production, and [68] further introduced the entropy flow vector and the entropy production density for it. In fact, the reversibility of $T_{\mathrm{A}} d S_{\mathrm{A}}=\delta Q_{\mathrm{A}}$ simply allows for such a possibility in principle, rather than the realistic occurrence of the reverse process, so we believe that the entropy-production treatment in [68] is inappropriate. As shown in Sec. VI.3, irreversibility and entropy production is a common feature for such (minimally coupled) modified gravities with a nontrivial effective gravitational coupling strength $\left(G_{\text {eff }} \neq\right.$ constant $)$ when their field equations are cast into the GR form $R_{\mu v}-$ $R g_{\mu \nu} / 2=8 \pi G_{\text {eff }} T_{\mu \nu}^{(\mathrm{eff})}$, and the time evolution of $G_{\text {eff }}$ causes irreversible energy dissipation and constitutes the only source of entropy production. 
[1] G W Gibbons, S W Hawking. Cosmological event horizons, thermodynamics, and particle creation. Phys. Rev. D 15, 2738 (1977).

Emil Mottola. Thermodynamic instability of de Sitter space. Phys. Rev. D 33, 1616 (1986).

P C W Davies. Cosmological horizons and the generalized second law of thermodynamics. Class. Quant. Grav. 4, L225-L228 (1987).

P C W Davies. Cosmological horizons and entropy. Class. Quant. Grav. 5, 1349 (1988).

[2] Rong-Gen Cai, Sang Pyo Kim. First law of thermodynamics and Friedmann Equations of Friedmann-Robertson-Walker universe. Journal of High Energy Physics 2005, 050 (2005). hep-th/0501055

[3] Ted Jacobson. Thermodynamics of spacetime: The Einstein equation of state. Phys. Rev. Lett. 75, 1260-1263 (1995). grqc/9504004

[4] T. Padmanabhan. Thermodynamical aspects of gravity: New insights. Rept. Prog. Phys. 73, 046901 (2010). arXiv:0911.5004

[5] J.M. Bardeen, B. Carter, S.W. Hawking. The four laws of black hole mechanics. Commun. Math. Phys. 31, 161-170 (1973).

Jacob D Bekenstein. Black holes and entropy. Phys. Rev. D 7, 2333-2346 (1973).

S.W. Hawking. Black hole explosions? Nature 248, 30-31 (1974).

[6] M. Akbar, Rong-Gen Cai. Thermodynamic behavior of Friedmann equations at apparent horizon of FRW universe. Phys. Rev. D 75, 084003 (2007). hep-th/0609128

[7] Rong-Gen Cai, Li-Ming Cao. Unified first law and the thermodynamics of the apparent horizon in the FRW universe. Phys. Rev. D 75, 064008 (2007). gr-qc/0611071

[8] M Akbar, Rong-Gen Cai. Thermodynamic behavior of field equations for $f(R)$ gravity. Phys. Lett. B 648, 243-248 (2007). gr-qc/0612089

[9] Rong-Gen Cai, Li-Ming Cao. Thermodynamics of apparent horizon in brane world scenario. Nucl. Phys. B 785, 135-148 (2007). hep-th/0612144

Ahmad Sheykhi, Bin Wang, Rong-Gen Cai. Thermodynamical properties of apparent horizon in warped DGP braneworld. Nucl. Phys. B 779, 1-12 (2007). hep-th/0701198

[10] Ahmad Sheykhi, Bin Wang, Rong-Gen Cai. Deep connection between thermodynamics and gravity in Gauss-Bonnet braneworlds. Phys. Rev. D 76, 023515 (2007). hep-th/0701261

[11] Kazuharu Bamba, Chao-Qiang Geng, Shinji Tsujikawa. Equilibrium thermodynamics in modified gravitational theories. Phys. Lett. B 688, 101-109 (2010). arXiv:0909.2159

[12] Rong-Gen Cai, Nobuyoshi Ohta. Horizon thermodynamics and gravitational field equations in Horava-Lifshitz gravity. Phys. Rev. D 81, 084061 (2010) arXiv:0910.2307

Qiao-Jun Cao, Yi-Xin Chen, Kai-Nan Shao. Clausius relation and Friedmann equation in FRW universe model. Journal of Cosmology and Astroparticle Physics 1005, 030 (2010). arXiv: 1001.2597

[13] Kazuharu Bamba, Chao-Qiang Geng, Shin'ichi Nojiri, Sergei D Odintsov. Equivalence of modified gravity equation to the Clausius relation. Europhys. Lett. 89, 50003 (2010). arXiv:0909.4397

[14] Ram Brustein. Generalized second law in cosmology from causal boundary entropy. Phys. Rev. Lett. 84, 2072 (2000). grqc/9904061

[15] M.R. Setare. Generalized second law of thermodynamics in quintom dominated universe. Phys. Lett. B 641, 130-133 (2006) hep-th/0611165

[16] M.R. Setare. Interacting holographic dark energy model and generalized second law of thermodynamics in non-flat universe. Journal of Cosmology and Astroparticle Physics 2007, 023 (2007). hep-th/0701242

K. Karami, A. Abdolmaleki. The generalized second law for the interacting new agegraphic dark energy in a non-flat FRW universe enclosed by the apparent horizon. Int. J. Theor. Phys. 50, 1656-1663 (2011). arXiv:0909.2427

Mubasher Jamil, Emmanuel N. Saridakis, M. R. Setare. Thermodynamics of dark energy interacting with dark matter and radiation. Phys. Rev. D 81, 023007 (2010). arXiv:0910.0822

K. Karami, S. Ghaffari. The generalized second law in irreversible thermodynamics for the interacting dark energy in a non-flat FRW universe enclosed by the apparent horizon. Phys. Lett. B 685, 115-119 (2010). arXiv:0912.0363

Surajit Chattopadhyay, Ujjal Debnath. Generalized second law of thermodynamics in presence of interacting DBI essence and other dark energies. Int. J. Mod. Phys. A25, 5557-5566 (2010). arXiv: 1008.1722

Surajit Chattopadhyay, Ujjal Debnath. Generalized second law of thermodynamics in presence of interacting tachyonic field and scalar(phantom)field. Can. J. Phys. 88, 933-938 (2010). arXiv: 1012.1784

[17] H. Mohseni Sadjadi. Generalized second law in modified theory of gravity. Phys. Rev. D 76, 104024 (2007). arXiv:0709.2435

[18] Shao-Feng Wu, Bin Wang, Guo-Hong Yang, Peng-Ming Zhang. The generalized second law of thermodynamics in generalized gravity theories. Class. Quant. Grav. 25, 235018 (2008). arXiv:0801.2688

[19] M. Akbar. Generalized second law of thermodynamics in extended theories of gravity. Int. J. Theor. Phys. 48, 2672-2678 (2009). arXiv:0808.3308

[20] Ahmad Sheykhi, Bin Wang. The Generalized second law of thermodynamics in Gauss-Bonnet braneworld. Phys. Lett. B 678, 434-437 (2009). arXiv:0811.4478

Ahmad Sheykhi, Bin Wang. Generalized second law of thermodynamics in warped DGP braneworld. Mod. Phys. Lett. A 25, 1199-1210 (2010). arXiv:0811.4477

[21] Mubasher Jamil, Emmanuel N Saridakis, M R Setare. The generalized second law of thermodynamics in Horava-Lifshitz cosmology. Journal of Cosmology and Astroparticle Physics 2010, 032 (2010). arXiv:1003.0876

[22] H. Mohseni Sadjadi. Cosmological entropy and generalized second law of thermodynamics in $F(R, G)$ theory of gravity. Europhys. Lett. 92, 50014 (2010). arXiv:1009.2941

[23] K. Karami, A. Abdolmaleki. Generalized second law of thermodynamics in $f(T)$-gravity. JCAP 1204, 007 (2012). arXiv:1201.2511

[24] A. Abdolmaleki, T. Najafi, K. Karami. Generalized second law of thermodynamics in scalar-tensor gravity. Phys. Rev. D 89, 104041 (2014). arXiv:1401.7549

[25] Ramon Herrera, Nelson Videla. The generalized second law of thermodynamics for interacting $f(R)$ gravity. Int. J. Mod. Phys. D 23, 1450071 (2014). arXiv:1406.6305

[26] Dongsu Bak, Soo-Jong Rey. Cosmic holography. Class. Quant. Grav. 17, L83 (2000). hep-th/9902173

[27] Abhay Ashtekar, Stephen Fairhurst, Badri Krishnan. Isolated horizons: Hamiltonian evolution and the first law. Phys. Rev. D 62, 104025 (2000). gr-qc/0005083 
[28] Sean A Hayward. General laws of black-hole dynamics. Phys. Rev. D 49, 6467-6474 (1994). gr-qc/9303006v3

[29] Tamara M Davis, Charles H Lineweaver. Expanding confusion: common misconceptions of cosmological horizons and the superluminal expansion of the universe. Publ. Astron. Soc. Austral. 21, 97-109 (2004). astro-ph/0310808

[30] Richard J Cook, M Shane Burns. Interpretation of the cosmological metric. Am. J. Phys. 77, 59-66 (2009). arXiv:0803.2701

[31] G Hinshaw, D Larson, E Komatsu, D N Spergel, C L Bennett, et al. Nine-year Wilkinson Microwave Anisotropy Probe (WMAP) observations: Cosmological parameter results . Astrophys. J. Suppl. 208, 19 (2013). arXiv:1212.5225

[32] S H Suyu, M W Auger, S Hilbert, P J Marshall, M Tewes, et al. Two accurate time-delay distances from strong lensing: Implications for cosmology. Astrophys. J. 766,70 (2013). arXiv: 1208.6010

[33] Éric Aubourg, Stephen Bailey, Julian E. Bautista, Florian Beutler, Vaishali Bhardwaj, et al. Cosmological implications of baryon acoustic oscillation (BAO) measurements. arXiv:1411.1074

[34] G. 't Hooft. Dimensional reduction in quantum gravity. grqc/9310026

Leonard Susskind. The World as a Hologram. J. Math. Phys. 36, 6377-6396 (1995). hep-th/9409089

Raphael Bousso. The holographic principle. Rev. Mod. Phys. 74, 825-874 (2002). hep-th/0203101

[35] T. Padmanabhan. The Holography of Gravity encoded in a relation between Entropy, Horizon area and Action for gravity. Gen. Rel. Grav. 34: 2029-2035 (2002). [gr-qc/0205090]

T. Padmanabhan. General relativity from a thermodynamic perspective. Gen. Rel. Grav. 46, 1673 (2014). [arXiv:1312.3253]

[36] Miao Li. A Model of holographic dark energy. Phys. Lett. B 603, 1 (2004). hep-th/0403127

[37] R.R. Caldwell, Rahul Dave, Paul J. Steinhardt. Cosmological imprint of an energy component with general equation of state. Phys. Rev. Lett. 80, 1582-1585 (1998). astro-ph/9708069

[38] P. J. E. Peebles, Bharat Ratra. The cosmological constant and dark energy. Rev. Mod. Phys. 75, 559-606 (2003). astro$\mathrm{ph} / 0207347$

T. Padmanabhan. Cosmological constant: The Weight of the vacuum. Phys. Rept. 380, 235-320 (2003) hep-th/0212290

Edmund J Copeland, M Sami, Shinji Tsujikawa. Dynamics of dark energy. International Int. J. Mod. Phys. D 15, 1753-1936 (2006). hep-th/0603057

Miao Li, Xiao-Dong Li, Shuang Wang, Yi Wang. Dark Energy. Commun. Theor. Phys. 56 525-604 (2011). arXiv:1103.5870

[39] R.R. Caldwell. A Phantom menace? Phys. Lett. B 545, 23-29 (2002). astro-ph/9908168

[40] Stephen W. Hawking, G.F.R. Ellis. The Large Scale Structure of Space-Time. Cambridge: Cambridge University Press, 1973.

[41] Ivan Booth. Black hole boundaries. Can. J. Phys. 83, 1073-1099 (2005). gr-qc/0508107v2

[42] Abhay Ashtekar, Badri Krishnan. Dynamical horizons: Energy, angular momentum, fluxes and balance laws. Phys. Rev. Lett. 89, 261101 (2002). gr-qc/0207080

[43] Valerio Faraoni. Cosmological apparent and trapping horizons. Phys. Rev. D 84, 024003 (2011). arXiv:1106.4427

[44] Charles W Misner, David H Sharp. Relativistic equations for adiabatic, spherically symmetric gravitational collapse. Phys. Rev. 136, B571-576 (1964).

Sean A Hayward. Gravitational energy in spherical symmetry. Phys. Rev. D 53, 1938-1949 (1996). gr-qc/9408002

[45] Stephen W Hawking . Gravitational radiation in an expanding universe. J. Math. Phys. 9, 598-604 (1968).
[46] Sean A Hayward. Unified first law of black-hole dynamics and relativistic thermodynamics. Class. Quant. Grav. 15, 3147-3162 (1998). gr-qc/9710089

[47] Rong-Gen Cai, Li-Ming Cao, Ya-Peng Hu. Hawking radiation of apparent horizon in a FRW universe. Class. Quant. Grav. 26, 155018 (2009). arXiv:0809.1554

[48] Yungui Gong, Bin Wang, Anzhong Wang. Thermodynamical properties of the Universe with dark energy. Journal of Cosmology and Astroparticle Physics (2007), 2007: 024. [grqc/0610151]

[49] Jacob D Bekenstein. Generalized second law of thermodynamics in black-hole physics. Phys. Rev. D 9, 3292-3300 (1974).

[50] Ivan Booth, Stephen Fairhurst. The first law for slowly evolving horizons. Phys. Rev. Lett. 92, 011102 (2004). gr-qc/0307087

Ivan Booth, Stephen Fairhurst. Isolated, slowly evolving, and dynamical trapping horizons: Geometry and mechanics from surface deformations. Phys. Rev. D 75, 084019 (2007). grqc/0610032

[51] Chao-Jun Feng, Xin-Zhou Li, Ping Xi. Global behavior of cosmological dynamics with interacting Veneziano ghost. JHEP 1205, 046 (2012). arXiv: 1204.4055

[52] Ricardo Garcia-Salcedo, Tame Gonzalez, Israel Quiros, Michael Thompson-Montero. QCD ghost dark energy cannot (even roughly) explain the main features of the accepted cosmological paradigm. Phys. Rev. D 88, 043008 (2013). arXiv:1301.6832

Surajit Chattopadhyay. Generalized second law of thermodynamics in $Q C D$ ghost $f(G)$ gravity. Astrophys. Space Sci. 352, 937-942 (2014). arXiv:1406.5142

[53] Rong-Gen Cai, Zhong-Liang Tuo, Hong-Bo Zhang, Qiping Su. Notes on ghost dark energy. Phys. Rev. D 84, 123501 (2011). arXiv: 1011.3212

[54] Arundhati Das, Surajit Chattopadhyay, Ujjal Debnath. Validity of generalized second law of thermodynamics in the logamediate and intermediate scenarios of the Universe. Found. Phys. 42, 266-283 (2012). arXiv:1104.2378

[55] Nairwita Mazumder, Subenoy Chakraborty. Does the validity of the first law of thermodynamics imply that the generalized second law of thermodynamics of the universe is bounded by the event horizon? Class. Quant. Grav. 26, 195016 (2009).

Nairwita Mazumder, Subenoy Chakraborty. Validity of the generalized second law of thermodynamics of the universe bounded by the event horizon in holographic dark energy model. Gen. Rel. Grav. 42, 813-820 (2010). arXiv:1005.3403

[56] Nairwita Mazumder, Subenoy Chakraborty. The Generalized second law of thermodynamics of the universe bounded by the event horizon and modified gravity theories. Int. J. Theor. Phys. 50, 251-259 (2011). arXiv:1005.5215

Nairwita Mazumder, Subenoy Chakraborty. Scalar-Tensor Theory of Gravity and Generalized Second Law of Thermodynamics on the Event Horizon. Astrophys. Space Sci. 332, 509-513 (2011). arXiv:1005.5217

[57] Shin'ichi Nojiri, Sergei D Odintsov. Introduction to modified gravity and gravitational alternative for dark energy. Int. J. Geom. Meth. Mod. Phys. 04, 115-146 (2007). hep-th/0601213 Shin'ichi Nojiri, Sergei D Odintsov. Unified cosmic history in modified gravity: from $F(R)$ theory to Lorentz non-invariant models. Phys. Rept. 505 59-144 (2011). arXiv:1011.0544 Timothy Clifton, Pedro G. Ferreira, Antonio Padilla, Constantinos Skordis. Modified gravity and cosmology. Phys. Rept. 513, 1-189 (2012). arXiv:1106.2476

Salvatore Capozziello, Mariafelicia De Laurentis. Extended Theories of Gravity. Phys. Rept. 509, 167-321 (2011). arXiv:1108.6266 
Kazuharu Bamba, Salvatore Capozziello, Shin'ichi Nojiri, Sergei D. Odintsov. Dark energy cosmology: the equivalent description via different theoretical models and cosmography tests. Astrophys. Space Sci. 342, 155-228 (2012). arXiv:1205.3421

[58] David W Tian, Ivan Booth. Lessons from $f\left(R, R_{c}^{2}, R_{m}^{2}, \mathscr{L}_{m}\right)$ gravity: Smooth Gauss-Bonnet limit, energy-momentum conservation, and nonminimal coupling. Phys. Rev. D 90, 024059 (2014). arXiv:1404.7823

[59] Antonio De Felice, Shinji Tsujikawa. $f(R)$ theories. Living Rev. Rel. 13, 3 (2010). arXiv:1002.4928

Salvatore Capozziello, Valerio Faraoni. Beyond Einstein Gravity: A Survey of Gravitational Theories for Cosmology and Astrophysics. Dordrecht: Springer, 2011.

[60] K S Stelle. Classical gravity with higher derivatives. Gen. Rel. Grav. 9, 353-371 (1978).

[61] R Jackiw, S Y Pi. Chern-Simons modification of general relativity. Phys. Rev. D 68, 104012 (2003). gr-qc/0308071

[62] C Brans, R H Dicke. Mach's principle and a relativistic theory of gravitation. Phys. Rev. 124, 925-935 (1961).

[63] Varun Sahni, Alexei Starobinsky. Reconstructing dark energy. Int. J. Mod. Phys. D 15, 2105-2132 (2006). astro-ph/0610026
[64] David Wenjie Tian, Ivan Booth. Friedmann equations from nonequilibrium thermodynamics of the Universe: A unified formulation for modified gravity. Phys. Rev. D 90, 104042 (2014). arXiv: 1409.4278

[65] Robert M Wald. Black hole entropy is the Noether charge. Phys. Rev. D 48, R3427-R3431 (1993). gr-qc/9307038

Ted Jacobson, Gungwon Kang, Robert C Myers. On black hole entropy. Phys. Rev. D 49, 6587-6598 (1994). gr-qc/9312023

Vivek Iyer, Robert M Wald. Some properties of the Noether charge and a proposal for dynamical black hole entropy. Phys. Rev. D 50, 846-864 (1994). gr-qc/9403028

[66] Tiberiu Harko. Thermodynamic interpretation of the generalized gravity models with geometry-matter coupling. Phys. Rev. D 90, 044067 (2014). arXiv:1408.3465

[67] Christopher Eling, Raf Guedens, Ted Jacobson. Nonequilibrium thermodynamics of spacetime. Phys. Rev. Lett. 96, 121301 (2006). arXiv:gr-qc/0602001

[68] Wang Gang, Liu Wen-Biao. Nonequilibrium thermodynamics of dark energy on cosmic apparent horizon. Commun. Theor. Phys. 52, 383-384 (2009). 